\title{
Two ultimate tests of constrained supersymmetry
}

\author{
Kamila Kowalska, Leszek Roszkowski ${ }^{1}$ and Enrico Maria Sessolo \\ National Centre for Nuclear Research, \\ Hoża 69, 00-681 Warsaw, Poland \\ E-mail: Kamila.Kowalska@fuw.edu.pl, L.Roszkowski@sheffield.ac.uk, \\ Enrico-Maria.Sessolo@fuw.edu.pl
}

ABSTRACT: We examine the prospects of using two alternative and complementary ways to explore the regions that are favored by global constraints in two simple unified supersymmetric models: the CMSSM and the NUHM. First, we consider $\operatorname{BR}\left(B_{s} \rightarrow \mu^{+} \mu^{-}\right)$, which has recently been for the first time measured by LHCb. In the CMSSM we show that ultimate, but realistic, improvement in the determination of the observable to about $5-10 \%$ around the Standard Model value would strongly disfavor the $A$-funnel region, while not affecting much the other favored regions. Second, we show that all the favored regions of the CMSSM will be, for the most part, sensitive to direct dark matter searches in future one-tonne detectors. A signal at low WIMP mass $(\lesssim 450 \mathrm{GeV})$ and low spin-independent cross section would then strongly favor the stau coannihilation region while a signal at higher WIMP mass $(\sim 800 \mathrm{GeV}$ to $\sim 1.2 \mathrm{TeV})$ would clearly point to the region where the neutralino is higgsino-like with mass $\sim 1 \mathrm{TeV}$. A nearly complete experimental testing of the CMSSM over multi-TeV ranges of superpartner masses, far beyond the reach of direct SUSY searches at the LHC, can therefore be achievable. In the NUHM, in contrast, similar favored regions exist but a sample study reveals that even a precise determination of $\mathrm{BR}\left(B_{s} \rightarrow \mu^{+} \mu^{-}\right)$would have a much less constraining power on the model, including the $A$-funnel region. On the other hand, this could allow one to, by detecting in one-tonne detectors a signal for $500 \mathrm{GeV} \lesssim m_{\chi} \lesssim 800 \mathrm{GeV}$, strongly disfavor the CMSSM.

KEYWORDS: Supersymmetry Phenomenology

ARXIV EPRINT: 1302.5956

\footnotetext{
${ }^{1}$ On leave of absence from the University of Sheffield, U.K.
} 


\section{Contents}

1 Introduction 1

$2 \operatorname{BR}\left(B_{s} \rightarrow \mu^{+} \mu^{-}\right)$in the MSSM 4

2.1 Application to the CMSSM 6

2.2 The $A$-funnel region vs $\operatorname{BR}\left(B_{s} \rightarrow \mu^{+} \mu^{-}\right) \quad 8$

3 Scanning methodology and constraints $\quad 10$

$\begin{array}{ll}3.1 \text { Experimental constraints } & 10\end{array}$

$\begin{array}{lll}\text { 3.1.1 Combination of CMS SUSY search limits } & 12\end{array}$

$\begin{array}{lll}3.2 & \text { Scanning tools and parameter ranges } & 14\end{array}$

$\begin{array}{llr}4 & \text { Results } & \mathbf{1 5}\end{array}$

$\begin{array}{ll}4.1 \text { The CMSSM } & 16\end{array}$

4.2 The NUHM 24

$\begin{array}{llr}5 & \text { Summary and conclusions } & 27\end{array}$

\section{Introduction}

In November 2012 the LHC reached the end of its current data collecting phase with the proton-proton beam at $\sqrt{s}=8 \mathrm{TeV}$. A huge amount of data was collected, allowing the CMS and ATLAS collaborations to reach an integrated luminosity of around $23 / \mathrm{fb}$ each, and $\mathrm{LHCb}$ of around $2.2 / \mathrm{fb}$. The performance of the detectors at the LHC and the effort of the experimental collaborations have been quite spectacular. The past year brought some experimental results whose crucial importance cannot be questioned, even though they still require further investigation and confirmation with larger amounts of data.

Most notably, on July 4, 2012, both the CMS and ATLAS collaborations announced a $5 \sigma$ discovery of a particle consistent with the Higgs boson predicted by the Standard Model (SM) based on the analysis of $4.9 / \mathrm{fb}$ of $p p$ collisions at $\sqrt{s}=7 \mathrm{TeV}[1,2]$. Both collaborations have recently updated their results, combining data from the $\sqrt{s}=7 \mathrm{TeV}$ and $\sqrt{s}=8 \mathrm{TeV}$ runs. The CMS value of the Higgs-like boson mass, $125.8 \pm 0.6 \mathrm{GeV}[3]$, is based on the analysis of the data corresponding to integrated luminosities of $5.1 / \mathrm{fb}$ at $\sqrt{s}=7 \mathrm{TeV}$ and up to $12.2 / \mathrm{fb}$ at $\sqrt{s}=8 \mathrm{TeV}$ in the $\gamma \gamma, Z Z, W W, \tau \tau$ and $b b$ decay channels. The ATLAS analysis combined approximately $4.8 / \mathrm{fb}$ of data at $\sqrt{s}=7 \mathrm{TeV}$ with $5.8 / \mathrm{fb}$ of data at $\sqrt{s}=8 \mathrm{TeV}$ in the same five channels, obtaining $125.2 \pm 0.7 \mathrm{GeV}$ [4].

On November 13, 2012 the LHCb Collaboration reported the first evidence of an excess in the rare decay $B_{s} \rightarrow \mu^{+} \mu^{-}$[5]. The measured value of the branching ratio, $\mathrm{BR}\left(B_{s} \rightarrow \mu^{+} \mu^{-}\right)=\left(3.2_{-1.2}^{+1.5}\right) \times 10^{-9}$, is consistent with the value predicted by the SM. 
This decay has been long considered as one of the best probes for new physics, and in particular for low-scale supersymmetry (SUSY), since SUSY contributions can be largely enhanced by the sixth power of $\tan \beta$, the ratio of the vacuum expectation values of the two Higgs doublets (see, e.g., [6-11] for some early studies).

The agreement of the recent measurement with the SM makes it potentially strongly constraining for the allowed parameter space of SUSY models. On the other side, the result still suffers from substantial experimental uncertainties - its current $2 \sigma$ upper bound is actually weaker than the previous 95\% confidence level (CL) exclusion limit $\mathrm{BR}\left(B_{s} \rightarrow \mu^{+} \mu^{-}\right)<4.5 \times 10^{-9}$ obtained earlier by the same collaboration [12].

Finally, on the front of direct SUSY searches, the $\sqrt{s}=7 \mathrm{TeV}$ and $\sqrt{s}=8 \mathrm{TeV}$ runs have significantly improved the limits on the masses of colored superpartners, allowing this way both CMS and ATLAS to exclude increasingly larger ranges of parameters of low-energy SUSY models. Currently the most constraining 95\% CL exclusion limits on the parameter space of the Constrained Minimal Supersymmetric Standard Model (CMSSM) comes from the ATLAS search for squarks and gluinos with jets and missing transverse energy in the final states, with $5.8 / \mathrm{fb}$ of data at $\sqrt{s}=8 \mathrm{TeV}$ [13]. A similar analysis by CMS based on $11.7 / \mathrm{fb}$ of data and using the kinematical variable $\alpha_{T}$ as a discriminator is slightly less constraining [14]. On the other hand, as we will show in this paper, the CMS razor analysis at $\sqrt{s}=7 \mathrm{TeV}$ with $4.4 / \mathrm{fb}$ [15] can be combined with the most recent $\alpha_{T}$ at $\sqrt{s}=8 \mathrm{TeV}$ [14] to produce a lower bound on the mass parameters of the CMSSM that, in the regions favored by the global constraints, is comparable to the current one from ATLAS.

In our recent global Bayesian analysis of the CMSSM [16] (as well as in several other recent global, Bayesian or $\chi^{2}$-based, analyses $\left.[17-25]\right),{ }^{1}$ it was shown or reiterated that, when combining through the likelihood function the $\mathrm{BR}\left(B_{s} \rightarrow \mu^{+} \mu^{-}\right)$bound from ref. [12], the Higgs mass, limits from direct SUSY searches, the relic density of dark matter (DM), an excess in the anomalous magnetic moment of the muon $(g-2)_{\mu}$, and other relevant constraints, four clearly identifiable regions of the $\left(m_{0}, m_{1 / 2}\right)$ plane, with $m_{0}$ and $m_{1 / 2}$ denoting the scalar and gaugino soft masses, respectively, remain favored by high posterior probability for both signs of the Higgs/higgsino mass parameter $\mu$ :

(a) at small $m_{0} \lesssim 400 \mathrm{GeV}$ and $600 \mathrm{GeV} \lesssim m_{1 / 2} \lesssim 1000 \mathrm{GeV}$, where the correct relic abundance is obtained via efficient neutralino-stau coannihilation [26] (staucoannihilation (SC) region hereafter). In this region the lightest bino-like neutralino as the lightest SUSY particle (LSP) is fairly light, $m_{\chi} \lesssim 450 \mathrm{GeV}$, and so is the lightest stop, $m_{\tilde{t}_{1}} \sim 1 \mathrm{TeV}$, hence the correct Higgs mass is achieved due to maximal stop mixing, $A_{t}^{2} / m_{\tilde{t}}^{2} \sim 6$;

\footnotetext{
${ }^{1} \mathrm{~A}$ note of caution is in order regarding a quantitative comparison of different analyses. First, Bayesian posterior high probability credible regions and $\chi^{2}$ confidence regions need not agree as they are based on two different concepts of probability. Secondly, even within the same statistical framework, numerical results often strongly depend on the values of input parameters used. For instance, the mass of the lightest Higgs boson in SUSY very sensitively depends on the exact value of the top quark pole mass, which is different in, e.g., [16] and [23].
} 
(b) at $1 \mathrm{TeV} \lesssim m_{1 / 2} \lesssim 2 \mathrm{TeV}$, where the cross-section for neutralino annihilation is enhanced by the $s$-channel resonance of the pseudoscalar $A$ Higgs boson [27] ( $A$-funnel (AF) region), with bino-like LSP in the mass range $350 \mathrm{GeV} \lesssim m_{\chi} \lesssim 700 \mathrm{GeV}$;

(c) for $m_{0} \gtrsim 3 \mathrm{TeV}, m_{0}>m_{1 / 2}$, in a strip of the $\left(m_{0}, m_{1 / 2}\right)$ plane along the border of the non-electroweak symmetry-breaking region, where the neutralino remains bino dominated but contains a non-negligible higgsino component [28, 29] (Focus Point/Hyperbolic Branch (FP/HB) region). In the FP/HB region we found [16] a significantly lower posterior probability primarily because it was difficult to obtain the correct mass of the Higgs boson. Also, this region is in considerable tension with current 90\% CL upper bound from XENON100 [30] on the spin-independent cross section $\sigma_{p}^{\text {SI }}$ of dark matter $(\mathrm{DM})$ scattering off xenon nuclei.

(d) in the multi- $\mathrm{TeV}$ regime $\left(m_{0} \gtrsim 4 \mathrm{TeV}, m_{1 / 2} \gtrsim 2 \mathrm{TeV}\right)$ there is a large region where the neutralino LSP is almost purely higgsino-like [20]. Its mass is almost constant, $m_{\chi} \approx \mu \simeq 1 \mathrm{TeV}$ (1TH region hereafter) so that the relic density constraint is easily satisfied, since for such a heavy higgsino LSP coannihilation is no longer effective.

A similar pattern holds also in the Non-Universal Higgs Model (NUHM), although at somewhat different locations. Specifically, the $1 \mathrm{TH}$ region can be found already at much lower mass scales, $m_{0} \lesssim 4 \mathrm{TeV}$ and $m_{1 / 2} \lesssim 2 \mathrm{TeV}$ [31]. (For an updated analysis including LHC data, see [23].)

Clearly, given such large mass scales most of the favored regions will remain beyond the reach of direct searches at the LHC. Only part of the SC and a small fraction of the AF and $\mathrm{FP} / \mathrm{HB}$ regions will be explored. It is therefore interesting to investigate the power of less direct ways of experimentally testing those regions, including projected sensitivities, on the most popular constrained SUSY models like the CMSSM or the NUHM. In this paper we will investigate two such observational venues: future measurements of $\mathrm{BR}\left(B_{s} \rightarrow \mu^{+} \mu^{-}\right)$ at the LHC and expected reach of direct search for DM through one-tonne detectors.

Regarding $\operatorname{BR}\left(B_{s} \rightarrow \mu^{+} \mu^{-}\right)$, in [16] it was also shown that the impact of the experimental upper bound (at that time) on $\mathrm{BR}\left(B_{s} \rightarrow \mu^{+} \mu^{-}\right)$was the strongest on the AF region where the SUSY contribution to the branching ratio is comparable to the SM one, while the other explored regions were less affected. A similar conclusion was reached in [32], where the impact of the new positive measurement of $\operatorname{BR}\left(B_{s} \rightarrow \mu^{+} \mu^{-}\right)$was for the first time investigated in the framework of the Constrained Next-to-Minimal Supersymmetric SM (CNMSSM), which also features similar favored regions when all the constraints are simultaneously taken into account. This points to an interesting relation between $\mathrm{BR}\left(B_{s} \rightarrow \mu^{+} \mu^{-}\right)$and the relic density constraint in the $\mathrm{AF}$ region.

As stated above, because the current experimental uncertainties are relatively large, the positive $\mathrm{LHCb}$ measurement of $\mathrm{BR}\left(B_{s} \rightarrow \mu^{+} \mu^{-}\right)$is actually somewhat less constraining for models of new physics predicting an enhancement of the observable than the previous exclusion bound. On the other hand, the systematic and statistical uncertainties will be greatly reduced when a larger amount of data comes, and are expected to ultimately achieve the level of $5 \%$. It is therefore interesting to investigate what impact such pro- 
jected sensitivities of $\mathrm{BR}\left(B_{s} \rightarrow \mu^{+} \mu^{-}\right)$will have on the favored regions of the CMSSM and the NUHM.

Our goal is twofold. First, we will present a Bayesian analysis of the current status of the CMSSM for a much broader range of input parameters than in [16]. We will apply the most recent experimental determinations of relevant input observables, most notably the Higgs boson mass and the top quark pole mass, in addition to the recent positive measurement of $\mathrm{BR}\left(B_{s} \rightarrow \mu^{+} \mu^{-}\right)$. We will show that, in the context of the CMSSM, the expected substantial reduction of the experimental and theoretical uncertainties in $\mathrm{BR}\left(B_{s} \rightarrow \mu^{+} \mu^{-}\right)$will have the potential to strongly disfavor basically the whole AF region. Secondly, we will show that the expected reach of direct search one-tonne DM detectors will be able to discriminate between the remaining two favored regions, the $\mathrm{SC}$ and the $1 \mathrm{TH}$ regions. On the other hand, we will show that, unfortunately, a similar conclusion cannot be reached in the NUHM because of the freedom in adjusting the pseudoscalar Higgs mass $m_{A}$ and the $\mu$ parameter. Still, in both models one should be able to distinguish between the $\mathrm{SC}$ and the $1 \mathrm{TH}$ regions. Furthermore, any DM signal indicative of the AF region would strongly disfavor the CMSSM.

Recently, ref. [33] analyzed the impact of the present measurement of $\mathrm{BR}\left(B_{s} \rightarrow \mu^{+} \mu^{-}\right)$ and its future status on random scans of the CMSSM and on the general MSSM (see also [34] for another recent analysis of this constraint in the MSSM), showing that a large fraction of the points generated would be excluded once the projected uncertainties in $\mathrm{BR}\left(B_{s} \rightarrow \mu^{+} \mu^{-}\right)$are considered. Our study is partly overlapping but differs in some important aspects: 1. Our analysis of the CMSSM is performed as a global Bayesian scan, where the constraints are applied simultaneously through the likelihood approach (with the exclusion of XENON100, as explained later). 2. Our main goal is to focus on the future ability to use $\mathrm{BR}\left(B_{s} \rightarrow \mu^{+} \mu^{-}\right)$to disfavor high probability regions of models with parameters unified at the scale of grand unification (GUT). As a consequence, we do not investigate the general MSSM, alongside to the CMSSM, but rather the NUHM model. 3. Unlike in [33], we will also discuss in the detail the implications of future direct searches of DM.

The paper is organized as follows. In section 2 we will demonstrate semi-analytically how $\operatorname{BR}\left(B_{s} \rightarrow \mu^{+} \mu^{-}\right)$shows a unique discriminating power over the $\mathrm{AF}$ region of the CMSSM. In section 3 we will describe our scanning methodology, and highlight the implementation of our statistical combination of CMS bounds on SUSY masses. In section 4 we will present our numerical results and discussion. Finally, we will give our Summary and Conclusions in section 5 .

\section{$2 \operatorname{BR}\left(B_{s} \rightarrow \mu^{+} \mu^{-}\right)$in the MSSM}

In this section we first quickly review the analytic form of $\mathrm{BR}\left(B_{s} \rightarrow \mu^{+} \mu^{-}\right)$in the MSSM and next analyze its implications for the AF region of the CMSSM and the NUHM.

The measurement of the branching ratio is a very good probe of new physics, since in the SM the decay rate is helicity suppressed, but can get significant contributions in SUSY. 
A general expression for the branching ratio is [35-38]

$\operatorname{BR}\left(B_{s} \rightarrow \mu^{+} \mu^{-}\right)=\frac{G_{F}^{2} \alpha_{\mathrm{em}}^{2} M_{B_{s}} \tau_{B_{s}}}{16 \pi^{3}}\left|V_{t b} V_{t s}^{*}\right|^{2} \sqrt{1-\frac{4 m_{\mu}^{2}}{M_{B_{s}}^{2}}}\left\{\left(1-\frac{4 m_{\mu}^{2}}{M_{B_{s}}^{2}}\right)\left|F_{S}\right|^{2}+\left|F_{P}+F_{A}\right|^{2}\right\}$,

where $M_{B_{s}}$ and $\tau_{B_{s}}$ are the $B_{s}$ mass and lifetime, and $F_{A}, F_{P}$ and $F_{S}$ are the axialvector, pseudo-scalar and scalar form factor, respectively. In the SM, $F_{S}$ and $F_{P}$ are highly suppressed by helicity conservation, and the only remaining term in the curly bracket in eq. (2.1) is $\left|F_{A}\right|^{2}$, where $F_{A}$ can be expressed in terms of the Wilson coefficient $C_{10}$, the muon mass $m_{\mu}$, and the $B_{s}$ decay constant $f_{B_{s}}, F_{A}=-i m_{\mu} f_{B_{s}} C_{10}$. The main source of theoretical uncertainty in calculating the SM value is the determination of $f_{B_{s}}$ by the lattice QCD groups. Ref. [39] estimates the $C P$-averaged branching ratio as $\mathrm{BR}\left(B_{s} \rightarrow \mu^{+} \mu^{-}\right)_{\mathrm{SM}}=(3.23 \pm 0.27) \times 10^{-9}$, while ref. [40] gives a slightly different value, $\mathrm{BR}\left(B_{s} \rightarrow \mu^{+} \mu^{-}\right)_{\mathrm{SM}}=(3.53 \pm 0.38) \times 10^{-9}$.

Notice that the theoretical calculation should be rescaled by the effects of $B_{s}-\bar{B}_{s}$ oscillations [41] in order to be compared with the experimentally measured value. In this study we will adopt the value given in [39] for the $C P$-averaged SM branching ratio and, following [41], take $\mathrm{BR}\left(B_{s} \rightarrow \mu^{+} \mu^{-}\right)_{\mathrm{SM}}=3.5 \times 10^{-9}$ for the value rescaled by the effects of $B_{s}-\bar{B}_{s}$ oscillations (time averaged). We differ here from [33] where $(3.87 \pm 0.46) \times 10^{-9}$ for the latter was used.

SUSY contributions to BR $\left(B_{s} \rightarrow \mu^{+} \mu^{-}\right)$become comparable to the SM when $F_{S}$ and $F_{P}$ are roughly of the same order as $F_{A}$. At the leading order (LO), in the framework of minimal flavor violation, the dominant SUSY terms in the Wilson coefficients are given by chargino-squark terms only and are proportional to $\tan ^{3} \beta$ [37].

Following the calculation and notation given in [37], one can write for $F_{P}$ and $F_{S}$

$$
F_{S, P} \simeq-\frac{i}{2} M_{B_{s}}^{2} f_{B_{s}} C_{S, P}
$$

where

$$
C_{S, P}=\mp \frac{m_{\mu}}{4 \sin ^{2} \theta_{W} M_{W}^{2}} \frac{\tan ^{3} \beta}{m_{A}^{2}} \mathcal{F}_{\mathrm{LO}} .
$$

The dominant contributions to $\mathcal{F}_{\mathrm{LO}}$ is given by the charginos and squarks in the loop,

$$
\begin{aligned}
\mathcal{F}_{\mathrm{LO}} & \simeq m_{\chi_{1}^{ \pm}} \sin \theta_{U}\left\{\sqrt{2} M_{W} \cos \theta_{V}\left[-D_{3}\left(\frac{m_{\tilde{c}_{L}}^{2}}{m_{\chi_{1}^{ \pm}}^{2}}\right)+D_{3}\left(\frac{m_{\tilde{t}_{1}}^{2}}{m_{\chi_{1}^{ \pm}}^{2}}\right) \cos ^{2} \theta_{t}+D_{3}\left(\frac{m_{\tilde{t}_{2}}^{2}}{m_{\chi_{1}^{ \pm}}^{2}}\right) \sin ^{2} \theta_{t}\right]\right. \\
& \left.-m_{t} \sin \theta_{V} \sin \theta_{t} \cos \theta_{t}\left[D_{3}\left(\frac{m_{\tilde{t}_{1}}^{2}}{m_{\chi_{1}^{ \pm}}^{2}}\right)-D_{3}\left(\frac{m_{\tilde{t}_{2}}^{2}}{m_{\chi_{1}^{ \pm}}^{2}}\right)\right]\right\} \\
& +(\operatorname{sgn} \mu) m_{\chi_{2}^{ \pm}} \cos \theta_{U}\left\{\sqrt{2} M_{W} \sin \theta_{V}\left[D_{3}\left(\frac{m_{\tilde{c}_{L}}^{2}}{m_{\chi_{2}^{ \pm}}^{2}}\right)-D_{3}\left(\frac{m_{\tilde{t}_{1}}^{2}}{m_{\chi_{2}^{ \pm}}^{2}}\right) \cos ^{2} \theta_{t}-D_{3}\left(\frac{m_{\tilde{t}_{2}}^{2}}{m_{\chi_{2}^{ \pm}}^{2}}\right) \sin ^{2} \theta_{t}\right]\right. \\
& \left.-m_{t} \cos \theta_{V} \sin \theta_{t} \cos \theta_{t}\left[D_{3}\left(\frac{m_{\tilde{t}_{1}}^{2}}{m_{\chi_{2}^{ \pm}}^{2}}\right)-D_{3}\left(\frac{m_{\tilde{t}_{2}}^{2}}{m_{\chi_{2}^{ \pm}}^{2}}\right)\right]\right\},
\end{aligned}
$$

where we assumed $\lambda_{22} \equiv V_{c b} V_{c s}^{*} /\left(V_{t b} V_{t s}^{*}\right) \simeq-\lambda_{33}=-1$, and neglected a term in $\lambda_{11} \equiv$ $V_{u b} V_{u s}^{*} /\left(V_{t b} V_{t s}^{*}\right) \simeq-10^{-2}$. 
The $D_{3}(x)$ are loop functions,

$$
D_{3}(x)=\frac{x \ln x}{1-x},
$$

and $\cos \theta_{U, V}$ and $\sin \theta_{U, V}$ are elements of the chargino mixing matrices defined such that $U M_{\chi^{ \pm}} V^{T}=\operatorname{diag}\left(m_{\chi_{1}^{ \pm}}, m_{\chi_{2}^{ \pm}}\right)$(See appendix B of [37] for notation).

For the purpose of this analysis we shall assume that the experimental uncertainty in $\mathrm{BR}\left(B_{s} \rightarrow \mu^{+} \mu^{-}\right)$will eventually, with about $50 / \mathrm{fb}$ of data at $\sqrt{s}=14 \mathrm{TeV}$, be reduced to about $5 \%[42]$. We will also assume that the theoretical uncertainty will reach the precision of $5 \%[43]$. Hence $\operatorname{BR}\left(B_{s} \rightarrow \mu^{+} \mu^{-}\right)_{\text {proj }}=(3.50 \pm 0.25) \times 10^{-9}$ with both theoretical and experimental uncertainties added in quadrature. Further, we will primarily assume that the current SM value will be confirmed by experimental measurements from LHC with the above precision, although we will discuss some possible deviations. In particular, we shall briefly discuss the case that the current $\mathrm{LHCb}$ central value is instead confirmed, $\mathrm{BR}\left(B_{s} \rightarrow \mu^{+} \mu^{-}\right)_{\text {proj }}=(3.20 \pm 0.23) \times 10^{-9}$ and the case when the assumed ultimate error will be twice as large.

\subsection{Application to the CMSSM}

In the CMSSM, and more generally in unified SUSY models, eqs. (2.1)-(2.5) can be greatly simplified thanks to relations between the different sparticles.

The first and the third line in the right-hand side of eq. (2.4) are always opposite in sign and cancel each other out to a good approximation. Since in the SC and AF regions the neutralino is strongly bino-dominated, it follows that $m_{\chi_{1}^{ \pm}} \simeq M_{2}$ and $m_{\chi_{2}^{ \pm}} \simeq \mu$, so that $\sin \theta_{U} \sin \theta_{V} \simeq 0$ and the fourth line in eq. (2.4) is dominant. In the $\mathrm{FP} / \mathrm{HB}$ and the $1 \mathrm{TH}$ regions, where the roles of $m_{\chi_{1}^{ \pm}}$and $m_{\chi_{2}^{ \pm}}$are interchanged, $\cos \theta_{U} \cos \theta_{V} \simeq 0$ so that the second term is thus dominant. In all of the favored regions, by remembering that $\sin \theta_{t} \cos \theta_{t} \simeq m_{t} A_{t} /\left(m_{\tilde{t}_{1}}^{2}-m_{\tilde{t}_{2}}^{2}\right)$ and that, for moderate to large $\tan \beta$, a change in $\operatorname{sgn} \mu$ implies a change in the $\operatorname{sign}$ of $\sin \theta_{U}$, as $\tan \theta_{U} \propto 1 /\left(\cos \beta M_{2}+\sin \beta \mu\right)$, one can recast eq. (2.4) as

$$
\mathcal{F}_{\mathrm{LO}} \simeq-\mu \mathcal{D}_{3} \frac{m_{t}^{2} A_{t}}{m_{\tilde{t}_{1}}^{2}-m_{\tilde{t}_{2}}^{2}},
$$

where $\mathcal{D}_{3}$ is given by differences of $D_{3}$ functions and is in general of order $0.1-0.3$.

In our numerical analysis we will use full calculations to higher order, given by the latest numerical codes, but we can use the above approximation to show how a projected better determination of $\mathrm{BR}\left(B_{s} \rightarrow \mu^{+} \mu^{-}\right)$can affect the four regions favored by the correct relic density, as explained above.

For choices of parameters typical of the SC region, $\mathcal{F}_{\mathrm{LO}}$ is the largest, driven up by large values of $\mu, \mu>1000 \mathrm{GeV}$, and by maximal $A_{t} / M_{\mathrm{SUSY}}$, which gives $\mathcal{D}_{3} \simeq 0.3$. On the other hand, as can be seen from eq. (2.3), the branching ratio is suppressed by moderate $\tan \beta$ values, $\tan \beta \sim 5-30$ typical for the SC region. The $\tan \beta$ dependence of $\operatorname{BR}\left(B_{s} \rightarrow \mu^{+} \mu^{-}\right)$for a point representative of the $\mathrm{SC}$ region $\left(m_{0}=226 \mathrm{GeV}, m_{1 / 2}=\right.$ $827 \mathrm{GeV}, A_{0}=-1375 \mathrm{GeV}$ ) is shown in figure 1(a). The solid blue line gives the case $\mu>0$ and the dashed red line the case $\mu<0$. The thick lines show values of $\tan \beta$ characteristic of 


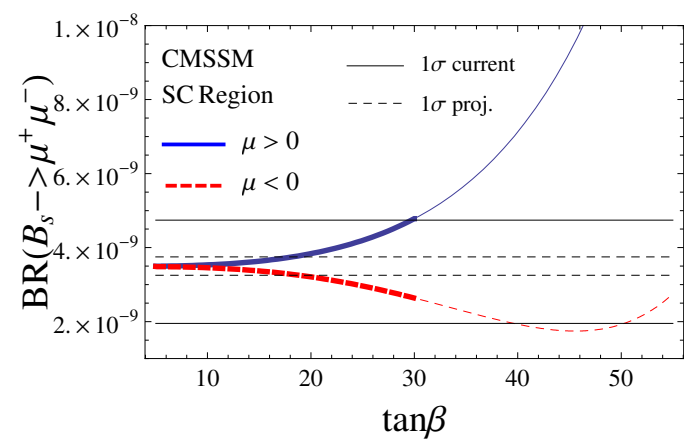

(a)

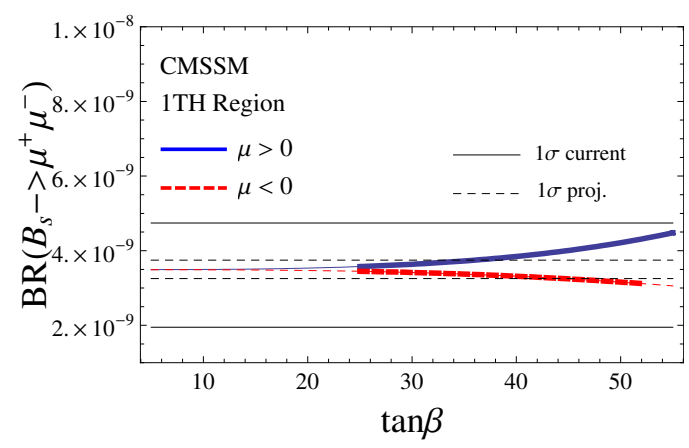

(c)

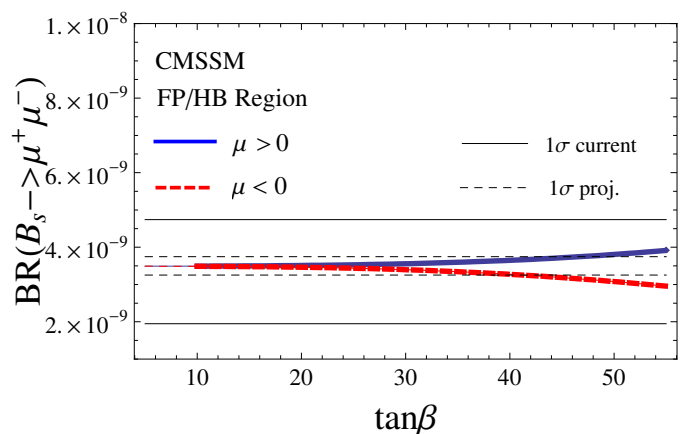

(b)

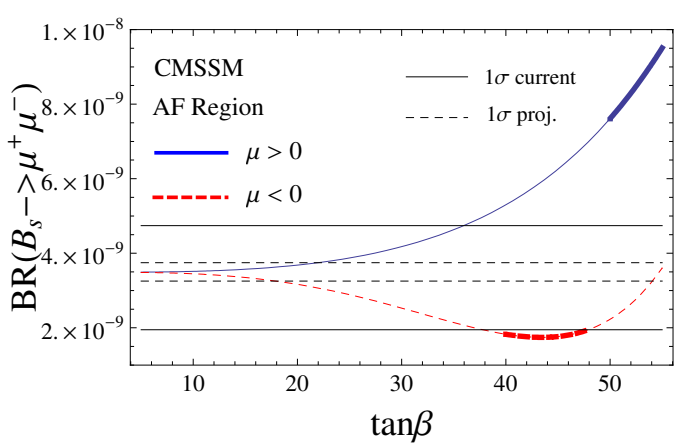

(d)

Figure 1. The dependence of $\mathrm{BR}\left(B_{s} \rightarrow \mu^{+} \mu^{-}\right)$on $\tan \beta$ in the CMSSM. Solid blue line: $\mu>0$; dashed red line: $\mu<0$. Solid horizontal lines: current $1 \sigma$ error on $\operatorname{BR}\left(B_{s} \rightarrow \mu^{+} \mu^{-}\right)$; dashed horizontal lines: projected error. The thick lines show the values of $\tan \beta$ typical of each region. (a) $\mathrm{SC}$ region. (b) $\mathrm{FP} / \mathrm{HB}$ region. (c) $1 \mathrm{TH}$ region. (d) AF region.

the $\mathrm{SC}$ region. The solid horizontal lines give the current $1 \sigma$ theoretical and experimental uncertainty on the measurement added in quadrature. The dashed horizontal lines denote our estimated $1 \sigma$ projected uncertainties added in quadrature.

In the $\mathrm{FP} / \mathrm{HB}$ region and, at large mass parameters, in the $1 \mathrm{TH}$ region, $\mathcal{F}_{\mathrm{LO}}$ is the smallest, since $A_{t} / M_{\mathrm{SUSY}}$ is minimal and $m_{\chi_{1}^{ \pm}} \approx \mu \lesssim 1 \mathrm{TeV}$. Moreover, the branching ratio is suppressed by large $m_{A}$, even if $\tan \beta$ can assume a wide range of values. We show in figure 1(b) the $\tan \beta$ dependence of $\mathrm{BR}\left(B_{s} \rightarrow \mu^{+} \mu^{-}\right)$for a point representative of the $\mathrm{FP} / \mathrm{HB}$ region $\left(m_{0}=3447 \mathrm{GeV}, m_{1 / 2}=866 \mathrm{GeV}, A_{0}=730 \mathrm{GeV}\right)$, and in figure $1(\mathrm{c})$, the same for a point representative of the $1 \mathrm{TeV}$ higgsino region $\left(m_{0}=7989 \mathrm{GeV}, m_{1 / 2}=\right.$ $2854 \mathrm{GeV}, A_{0}=-767 \mathrm{GeV}$ ). The color code is the same as in figure 1(a).

Finally, but most importantly for the purpose of this paper, in the AF region $\tan \beta$ has to be large in order to yield the correct $\Omega_{\chi} h^{2}$, as we will explain in the following subsection. Thus, the measured value of $\mathrm{BR}\left(B_{s} \rightarrow \mu^{+} \mu^{-}\right)$becomes important in constraining the parameter space ( $\mu$ and $m_{A}$ are comparable to the SC region, while stop mixing is not as large, so that $\left.\mathcal{D}_{3} \sim 0.15-0.2\right)$. In figure $1(\mathrm{~d})$ we again indicate with thick lines the ranges of $\tan \beta$, for both signs of $\mu$, which give $\Omega_{\chi} h^{2}$ within $1 \sigma$ (theoretical + experimental uncertainties added in quadrature) of the central value. The difference in the allowed values 
of $\tan \beta$ for different $\operatorname{sgn} \mu$ is a feature of the AF region, and was already observed in [16]. We will explain this in the next subsection. Notice also that, for $\mu<0$ the calculated value of $\mathrm{BR}\left(B_{s} \rightarrow \mu^{+} \mu^{-}\right)$is more than $1 \sigma$ below the SM value, since the form factors $F_{P}$ and $F_{A}$ undergo destructive interference, and one is left with a small value of $F_{S}$.

\subsection{The $A$-funnel region vs $\mathbf{B R}\left(B_{s} \rightarrow \mu^{+} \mu^{-}\right)$}

The AF region is particularly sensitive to the determination of $\mathrm{BR}\left(B_{s} \rightarrow \mu^{+} \mu^{-}\right)$because at the LO the relic density there depends mainly on the same parameters, $m_{A}$ and $\tan \beta$; see eq. (2.3).

AF annihilation occurs when the mass of the pseudoscalar $A$ is close $2 m_{\chi}$, so the lightest neutralino can efficiently annihilate into SM fermions (mostly $b$-quarks) through the $s$-channel exchange of $A$. For an almost pure bino LSP (higgsino components of order $10^{-2}$ or less) and $\tan \beta$ in the range $20-60$ (for lower values the channel $\chi \chi \rightarrow Z h$ becomes dominant) one obtains [27]

$$
\Omega_{\chi} h^{2} \approx \frac{3 \times 10^{-27} \mathrm{~cm}^{3} / \mathrm{s}}{\langle\sigma v\rangle},
$$

where

$$
\sigma v \approx \frac{\text { const }}{m_{\chi}^{2}} \frac{\tan ^{2} \beta}{\left(4-m_{A}^{2} / m_{\chi}^{2}\right)^{2}+\left(\Gamma_{A} m_{A} / m_{\chi}^{2}\right)^{2}}\left(1+\frac{v^{2}}{4}\right),
$$

with the $A$ width being, for $\mu>0(\mu<0), \Gamma_{A} \approx 1.3(2.0) \times 10^{-5} m_{A} \tan ^{2} \beta$. The constant in eq. (2.8) depends moderately on kinematical factors, on the neutralino composition, and on $\tan \beta$. For masses given in $\mathrm{GeV}$, its value is $\sim 10^{-25} \mathrm{~cm}^{3} \mathrm{GeV}^{2} / \mathrm{s}$. The correct relic density is generally achieved for a difference $\left|m_{A}-2 m_{\chi}\right|$ not exceeding $100 \mathrm{GeV}$ [44].

In the CMSSM with bino-like DM, $m_{A}$ can in principle be close to $2 m_{\chi}$ for wide ranges of $m_{0}$ and $m_{1 / 2}$, if the value of $\tan \beta$ is properly adjusted: the mass of a bino-like LSP is approximately given by $m_{\chi} \approx 0.44 m_{1 / 2}$ while $m_{A}=0.72 m_{1 / 2} \times f\left(\frac{50}{\tan \beta}\right)$, where $f(x)$ is a monotonically increasing function of $x$, and $f(1)=1$. As a consequence, for large $\tan \beta$, with increasing $m_{1 / 2} 2 m_{\chi}$ increases faster than $m_{A}$. Hence, in order to get the resonance for larger $m_{1 / 2}$, one needs to assume smaller $\tan \beta$. However, this does not mean that the correct relic density can always be obtained since, as eq. (2.8) shows, even for $m_{A} \simeq 2 m_{\chi}$ the cross section becomes suppressed with increasing neutralino mass for any given $\tan \beta$.

In figure 2 the green bands show the regions of the $\left(m_{0}, m_{1 / 2}\right)$ plane over which the condition $m_{A}=2 m_{\chi}$ is satisfied for fixed $\tan \beta$ and $-10 \mathrm{TeV} \leq A_{0} \leq 10 \mathrm{TeV}$. Figure 2 (a) shows the case $\mu>0$ and figure 2(b) $\mu<0$. Note that, this is achieved when, for the same $\tan \beta, m_{1 / 2}$ is slightly smaller for negative $\mu$ than for positive $\mu$. The reason lies in a one-loop tadpole contribution to the effective potential of the model [45]. The corrections due to sfermions, charginos and neutralinos explicitly depend on $\operatorname{sgn} \mu$, leading to positive (negative) contribution to $m_{A}^{2}$ for $\mu>0(\mu<0)$. Therefore, when the other parameters of the model are left unchanged, $m_{A}$ is slightly smaller for negative $\mu$. For the same reason, values of $\tan \beta$ larger than 50 cannot be obtained for negative $\mu$ since they would lead to $m_{A}^{2}<0$ and no electro-weak symmetry breaking.

Using eqs. (2.7) and (2.8), one can now calculate the ranges of $m_{1 / 2}$ that for a given $\tan \beta$ would allow to obtain the correct relic density (within $1 \sigma$ of the experimental central 


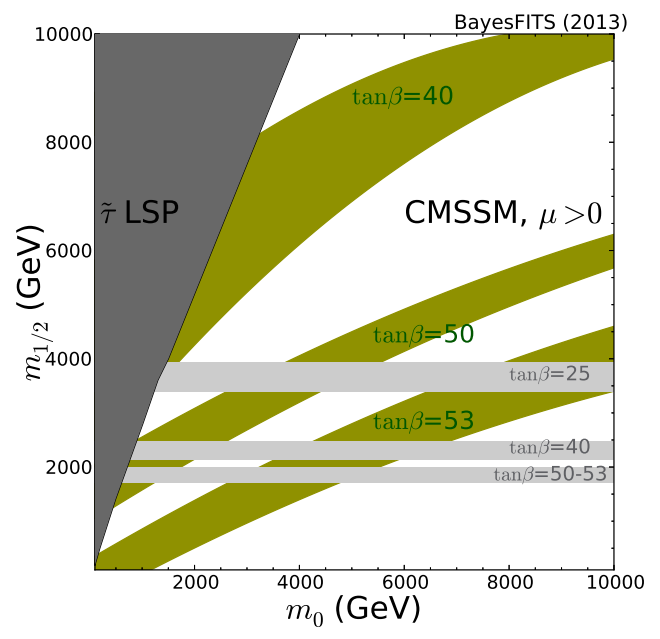

(a)

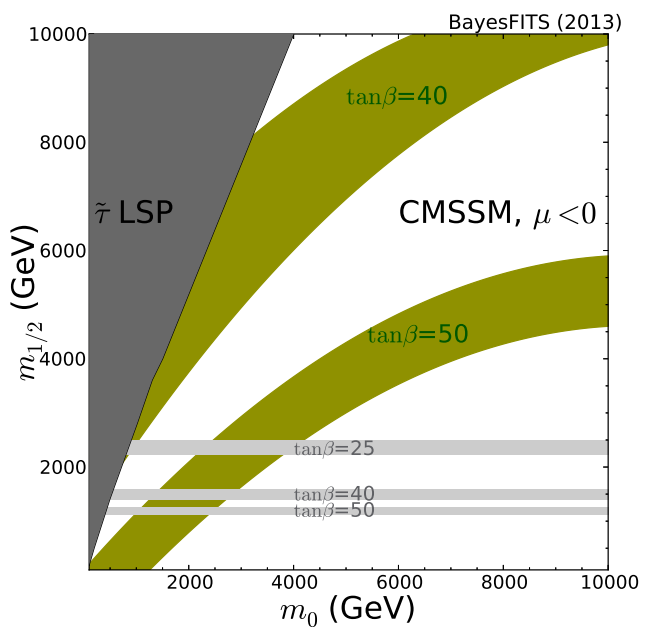

(b)

Figure 2. Green bands: the regions of the CMSSM where $m_{A}=2 m_{\chi}$ for $-10 \mathrm{TeV} \leq A_{0} \leq 10 \mathrm{TeV}$. Light gray bands: the regions where $\sigma v$ for bino-like DM gives the correct $\Omega_{\chi} h^{2}$ through $A$-resonant annihilation. Dark gray: the neutralino is not the LSP. (a) $\mu>0$. (b) $\mu<0$.

value) when $m_{A}=2 m_{\chi}$. We show them in figures $2(\mathrm{a})$ and $2(\mathrm{~b})$ as gray horizontal stripes. One can see that the AF region of the CMSSM is confined to a relatively small part of the $\left(m_{0}, m_{1 / 2}\right)$ plane, where the green and the grey bands of the same $\tan \beta$ intersect. This is also true in the more realistic case where $m_{A}$ and $2 m_{\chi}$ are within $100 \mathrm{GeV}$ from one another, as is confirmed by numerical scans. As a consequence, $\tan \beta$ is also constrained in the $\mathrm{AF}$ region: it can take values in the range $48-55$ for positive $\mu$, and $38-50$ for negative $\mu$. This is the ranges we highlighted in boldface in figure $1(\mathrm{~d})$.

One can also see in figure 1(d) that the values of $m_{A}$ and $\tan \beta$ typical of the AF region are the ones that show most tension with the current measurement of $\operatorname{BR}\left(B_{s} \rightarrow \mu^{+} \mu^{-}\right)$. Moreover, it is clear that future, more precise, measurements of the branching ratio will have the potential to exclude a broad range of the $\left(m_{0}, m_{1 / 2}\right)$ parameter space corresponding to the AF region.

In the NUHM the situation is quite different. The additional soft mass parameters in the Higgs sector $m_{H_{u}}$ and $m_{H_{d}}$ can be traded, through conditions of electroweak symmetry breaking (see, e.g., [31]), for $m_{A}$ and $\mu$ :

$$
\begin{aligned}
\mu^{2} & =\frac{m_{H_{d}}^{2}-m_{H_{u}}^{2} \tan ^{2} \beta}{\tan ^{2} \beta-1}-\frac{1}{2} M_{Z}^{2}, \\
m_{A}^{2} & =m_{H_{d}}^{2}+m_{H_{u}}^{2}+2 \mu^{2},
\end{aligned}
$$

which can be adjusted to satisfy the resonance condition, independently of $\tan \beta$, for much wider ranges of both $m_{0}$ and $m_{1 / 2}$. Therefore, in the NUHM the AF region giving the correct relic density is not as well localized and occupies a wide part of the parameter space. Also $\tan \beta$ is now allowed to assume a much wider range of input values, which is 
crucial from the point of view of satisfying the $\mathrm{BR}\left(B_{s} \rightarrow \mu^{+} \mu^{-}\right)$constraint. We will come back to this point later.

\section{Scanning methodology and constraints}

In order to examine the impact of the most recent constraints, including $\mathrm{BR}\left(B_{s} \rightarrow \mu^{+} \mu^{-}\right)$, on the parameter space of the CMSSM and the NUHM we use the Bayesian approach. We follow the procedure outlined in detail in refs. $[16,46,47]$. Our goal is to map out the $68 \%$ and $95 \%$ credible regions of $p(m \mid d)$, the posterior probability density function (pdf), given by Bayes' theorem,

$$
p(m \mid d)=\frac{p(d \mid \xi(m)) \pi(m)}{p(d)},
$$

where $p(d \mid \xi(m)) \equiv \mathcal{L}$ is the likelihood function, which describes the probability of obtaining the data $d$ given the computed value of some observable $\xi(m)$, which is a function of the model's parameters $m . \mathcal{L}$ incorporates the information about the constraints, as well as their experimental and theoretical uncertainties. Prior probability $\pi(m)$ encodes assumed range and distribution of $m$. Finally, $p(d)$ is the evidence and is a normalization constant as long as only one model is considered, but serves as a comparative measure for different models or scenarios.

Bayes' theorem provides an efficient and natural procedure for drawing inferences on a subset of $r$ specific model parameters (including nuisance parameters), or observables, or a combination of both, which we collectively denote by $\psi_{i}$. They can be obtained through marginalization of the full posterior pdf, carried out as

$$
p\left(\psi_{i=1, \ldots, r} \mid d\right)=\int p(m \mid d) d^{n-r} m,
$$

where $n$ is the total number of input parameters.

\subsection{Experimental constraints}

The central object in our analysis is the likelihood function as the place where theoretical predictions are compared with experimental data. The constraints that we include in the current analysis are listed in table 1. As a rule, following the procedure developed earlier [53], we implemented positive measurements through a Gaussian likelihood, in which the experimental and theoretical uncertainties were added in quadrature. For the Higgs mass, we used the most recent CMS determination of its central value and experimental uncertainty, as it is in perfect agreement with the determination obtained by ATLAS at the end of the $\sqrt{s}=8 \mathrm{TeV}$ run. The theoretical uncertainty was estimated to be $3 \mathrm{GeV}[16,54]$.

As stated above, for $\mathrm{BR}\left(B_{s} \rightarrow \mu^{+} \mu^{-}\right)$we considered two cases:

1. The current measurement at LHCb, for which we adopted a theoretical uncertainty of $10 \%$ of the measured value (see next-to-bottom row in table 1), in agreement with [40] once the uncertainty due to the top pole mass $(\sim 1 \%)$ is subtracted. We do so because in our scans the top mass is one of the nuisance parameters and the effect of varying it is included parametrically. 


\begin{tabular}{|l|l|l|l|l|}
\hline Measurement & Mean or Range & Error: (Exp., Th.) & Distribution & Ref. \\
\hline Combination of: & & & & \\
CMS razor $4.4 / \mathrm{fb}, \sqrt{s}=7 \mathrm{TeV}$ & See text & See text & Poisson & {$[15]$} \\
CMS $\alpha_{T} 11.7 / \mathrm{fb}, \sqrt{s}=8 \mathrm{TeV}$ & See text & See text & Poisson & {$[14]$} \\
\hline$m_{h}$ by CMS & $125.8 \mathrm{GeV}$ & $0.6 \mathrm{GeV}, 3 \mathrm{GeV}$ & Gaussian & {$[3]$} \\
$\Omega_{\chi} h^{2}$ & 0.1120 & $0.0056,10 \%$ & Gaussian & {$[48]$} \\
$\delta(g-2)_{\mu}^{\mathrm{SUS}} \times 10^{10}$ & 28.7 & $8.0,1.0$ & Gaussian & {$[49,50]$} \\
$\mathrm{BR}\left(\bar{B} \rightarrow X_{s} \gamma\right) \times 10^{4}$ & 3.43 & $0.22,0.21$ & Gaussian & {$[51]$} \\
$\mathrm{BR}\left(B_{u} \rightarrow \tau \nu\right) \times 10^{4}$ & 1.66 & $0.33,0.38$ & Gaussian & {$[52]$} \\
$\Delta M_{B_{s}}$ & $17.719 \mathrm{ps}^{-1}$ & $0.043 \mathrm{ps}{ }^{-1}, 2.400 \mathrm{ps}^{-1}$ & Gaussian & {$[49]$} \\
$\sin ^{2} \theta_{\mathrm{eff}}$ & 0.23116 & $0.00012,0.00015$ & Gaussian & {$[49]$} \\
$M_{W}$ & 80.385 & $0.015,0.015$ & Gaussian & {$[49]$} \\
\hline $\mathrm{BR}\left(B_{s} \rightarrow \mu^{+} \mu^{-}\right)_{\text {current }} \times 10^{9}$ & 3.2 & $+1.5-1.2,10 \%(0.32)$ & Gaussian & {$[5]$} \\
$\mathrm{BR}\left(B_{s} \rightarrow \mu^{+} \mu^{-}\right)_{\text {proj }} \times 10^{9}$ & $3.5\left(3.2^{*}\right)$ & $0.18\left(0.16^{*}\right), 5 \%\left[0.18\left(0.16^{*}\right)\right]$ & Gaussian & {$[5]$} \\
\hline
\end{tabular}

${ }^{*}$ We will also consider the case of projected uncertainties around the current measured central value.

Table 1. The experimental constraints that we apply to constrain model parameters.

2. The projected 'best-case' scenario for the determination of $\mathrm{BR}\left(B_{s} \rightarrow \mu^{+} \mu^{-}\right)$, where the experimental and theoretical uncertainties are both reduced to $5 \%$ of the measured value (see bottom row in table 1), as explained in section 2. In addition, as a sensitivity test, we considered both the case where the measurement will be narrowed down to the time-averaged SM value, $3.5 \times 10^{-9}$, and the case where the current central LHCb experimental value, $3.2 \times 10^{-9}$, will be confirmed by future sensitivities. This second case can in principle improve the fit for the AF region in the $\mu<0$ case, since the branching ratio there assumes values more than $1 \sigma$ below the SM determination (see figure 1(d) and [16]). Finally, we will double the assumed error around the SM value, again as a sensitivity test.

Following the procedure already adopted in our previous papers, we did not include the XENON100 upper bound explicitly in the likelihood function. The theory uncertainties are very large (up to a factor of 10) and strongly affect the impact of the experimental limit on the parameter space. The main source of error (the so-called $\Sigma_{\pi N}$ term [55]) arises from different, and in fact partly incompatible, results following from different calculations based on different assumptions and methodologies. Such uncertainties do not follow a particular statistical distribution, and are not well suited for inclusion in a likelihood function. Moreover, we showed in a previous publication [47] that, when smearing out the XENON100 limit with a theoretical uncertainty of order ten times the given value of $\sigma_{p}^{\mathrm{SI}}$ the effect on the posterior is negligible for regions of parameter that appear up to one order of magnitude above (and below) the experimental limit. However, even if we do not include the XENON100 bound in the likelihood, below we shall comment on its possible effects on the posterior pdf.

The likelihood for limits from direct SUSY searches deserves a more detailed explanation, which we give in the following subsection. 


\subsubsection{Combination of CMS SUSY search limits}

In previous work $[16,46]$ we presented a methodology for deriving approximate but accurate likelihood functions for two of the direct SUSY searches with all-hadronic final states at CMS: $\alpha_{T}$ (ref. [46]) and razor (ref. [16]). Our approximation correctly reproduced the 95\% CL exclusion bounds of those searches in the $\left(m_{0}, m_{1 / 2}\right)$ plane. In [32] we then showed that the same procedure for the razor search could be extended to the CNMSSM.

The likelihood maps were developed through a step-by-step procedure which included generation of the SUSY signal at the scattering level with PYTHIA6.4 [56] and a simulation of the CMS detector response with PGS4 [57] to calculate the efficiency once the kinematic cuts were applied. The obtained signal yields were finally statistically compared to the publicly available observed and background yields of the searches to construct the likelihood map.

As mentioned in the Introduction, the most constraining limit for the CMSSM presently comes from the ATLAS search for squarks and gluinos with jets and missing transverse energy in the final states, with $5.8 / \mathrm{fb}$ of data at $\sqrt{s}=8 \mathrm{TeV}$ [13]. The recent limits produced by the CMS Collaboration with comparable or larger luminosity [14] are slightly weaker. On the other hand, riding on our accurate method for constructing the likelihood function for all-hadronic SUSY search limits with the information provided by the CMS Collaboration, we are in a position of deriving an approximate statistical combination of the CMS searches at $\sqrt{s}=7 \mathrm{TeV}$ and $\sqrt{s}=8 \mathrm{TeV}$.

We prefer to follow this procedure rather than taking the ATLAS limit as a hard cut (as recently done, e.g., in [23, 58]) for one important reason. Most recent analyses of the CMSSM have pointed out that the region of parameter space which provides the best fit to the constraints (particularly the Higgs mass) is the SC region. Since this is the region directly adjacent to the exclusion bounds, accurate modelling of the likelihood function becomes important.

In what follows we briefly summarize the methodology adopted for the razor in our previous papers, since it will be used again here. We then proceed to statistically combining it with the most recent CMS $\alpha_{T}$ search to update our exclusion bound.

Razor 4.4/fb, $\sqrt{s}=7 \mathrm{TeV}$. The CMS razor search, based on $4.4 / \mathrm{fb}$ of $\sqrt{s}=7 \mathrm{TeV}$ data, found no excess of events over the SM prediction. In deriving the likelihood map for the razor analysis we followed the CMS procedure described in [15]. All accepted events were divided into 38 separate bins in the two-dimensional space of the razor variables $R^{2}$ and $M_{R}$, and the likelihood of observing a certain number events in a given bin was defined as a Poisson distribution convolved with a Gaussian or log-normal function that would take care of the predicted error on the background yields. The details of our analysis can be found in $[16]$.

$\boldsymbol{\alpha}_{\boldsymbol{T}} \mathbf{1 1 . 7} / \mathrm{fb}, \sqrt{\boldsymbol{s}}=\mathbf{8} \mathbf{T e V}$. The CMS $\alpha_{T}$ search, performed with $11.7 / \mathrm{fb}$ of data based on $\sqrt{s}=8 \mathrm{TeV} p p$ collisions, shows no significant deviation from the SM prediction [14]. In deriving the likelihood map we followed closely the CMS procedure and our methodology presented in $[46,47]$. The accepted events were divided into 8 separate boxes, according 


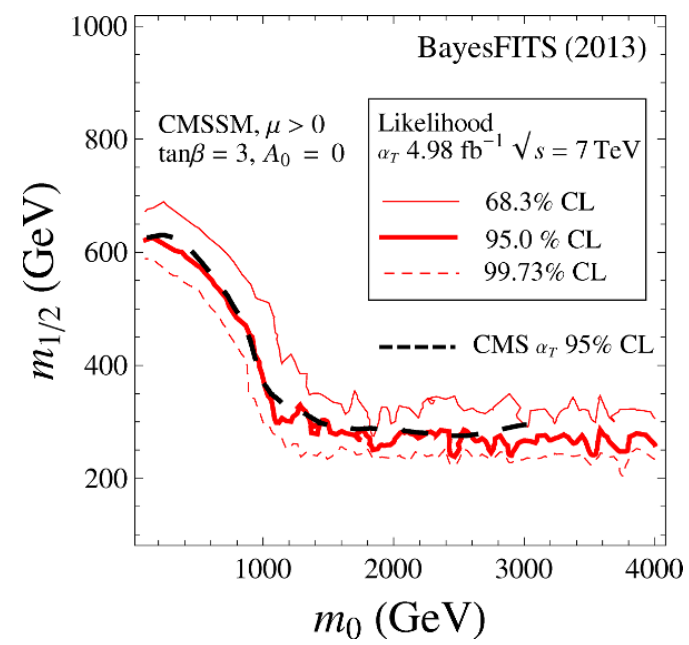

(a)

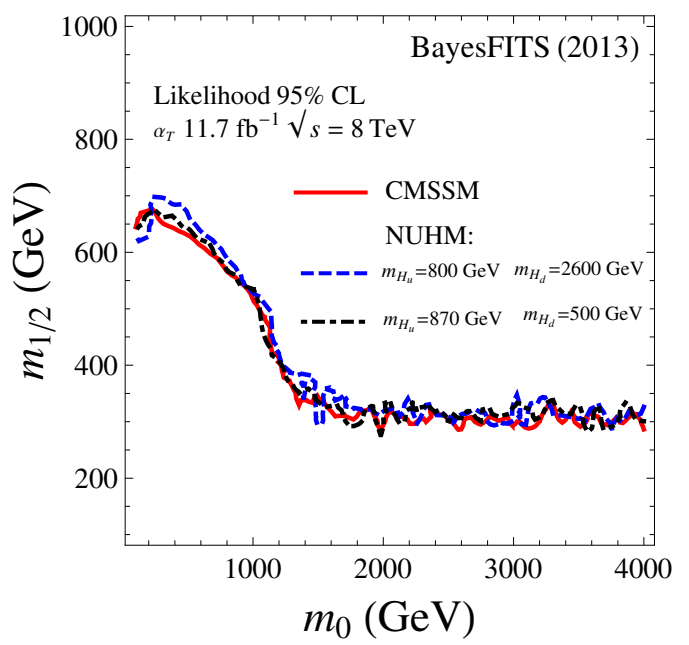

(b)

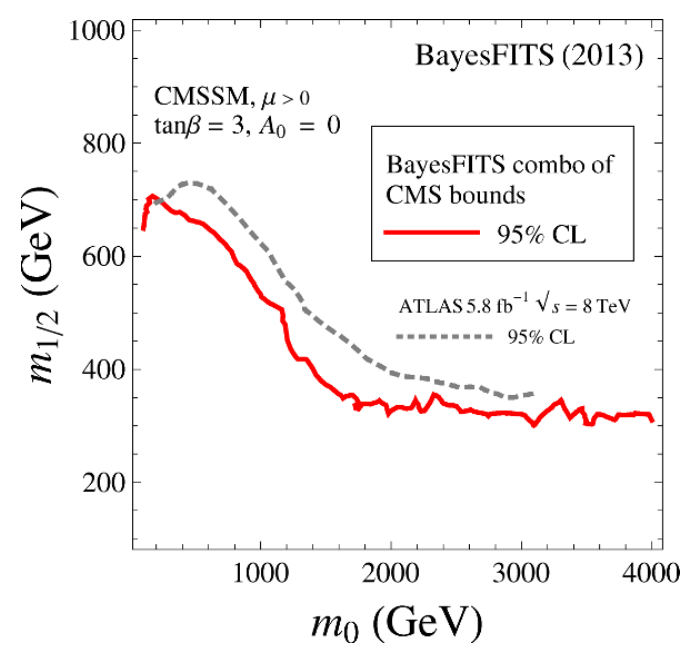

(c)

Figure 3. (a) The $68.3 \%$ CL (red solid thin), $95.0 \%$ CL (red solid thick), and 99.7\% CL (red dashed thin) exclusion bounds for the CMSSM from our approximation of the $\alpha_{T}$ likelihood $(\sqrt{s}=7 \mathrm{TeV}, \sim$ $5 / \mathrm{fb}$ ) compared to the original CMS 95\% CL exclusion bound (dashed black). (b) 95\% CL exclusion bound (solid red) for the CMSSM from our approximation of the $\alpha_{T}$ likelihood $(\sqrt{s}=8 \mathrm{TeV}$, $\sim 12 / \mathrm{fb}$ ) compared with the bounds obtained for the NUHM, when $m_{H_{u}}<m_{H_{d}}$ (dashed blue) and $m_{H_{d}}<m_{H_{u}}$ (dot-dashed black). (c) $95 \%$ CL exclusion bound (solid red) for the CMSSM from our combination of CMS searches (solid red) compared to the current ATLAS bound (dotted gray).

to the number of jets originating from $b$-quarks, $n_{b}=0,1,2,3$ or $\geq 4$ and to the number of reconstructed jets per event, $2 \leq n_{j} \leq 3$ and $n_{j} \geq 4$. In every box, the events were classified based on the value of the variable $H_{T}$, defined as the sum of all jets' transverse energies. The likelihood for observing $o_{i}$ events in the $i$-th bin, given the known number of the expected events $s_{i}$, and the number of the expected SM background events $b_{i}$, is given by a Poisson distribution convolved with a Gaussian, to account for the predicted error 
on the background yield. The ranges of $H_{T}$ in every bin, together with the corresponding numbers of the observed events, expected background events, and errors on the expected background yield provided by the CMS Collaboration, are given in [59].

Since the CMS Collaboration has not provided for this search the official 95\% CL exclusion bound in the CMSSM, we validated our likelihood map procedure for the $\alpha_{T}$ analysis with the official $\alpha_{T} 4.98 / \mathrm{fb}, \sqrt{s}=7 \mathrm{TeV}$ contour given in [60]. In figure 3(a) we show the comparison between our simulation and the official plot. As one can see, we obtained very good agreement. In figure $3(\mathrm{~b})$ we show our $95 \%$ CL contour for the $\alpha_{T} 11.7 / \mathrm{fb}, \sqrt{s}=8 \mathrm{TeV}$ as a solid red line. It is a big advantage of the likelihood map methodology that it allows one to derive likelihood functions for SUSY searches even where the official limits are not available.

Furthermore, we also show in figure 3(b) that the derived exclusion limit can be applied not only to the CMSSM, but also to the NUHM. The exclusion bounds obtained for two different choices of the parameters $m_{H_{u}}$ and $m_{H_{d}}$ (shown in dashed blue and dot-dashed black) do not differ from the CMSSM one. The reason is that the soft masses of the Higgs sector enter the one-loop renormalization group equations of the first two generation quarks only by the terms multiplied by the Yukawa couplings, and therefore are strongly suppressed, while the term proportional to the difference $\left(m_{H_{u}}^{2}-m_{H_{d}}^{2}\right)$ is multiplied by the factor $g_{1}^{2} / 10$ and is also negligible, unless the mass difference is very large. The NUHM exclusion limits shown at figure $3(\mathrm{~b})$ correspond precisely to the choice of parameters that would maximize the difference $\left|m_{H_{u}}^{2}-m_{H_{d}}^{2}\right|$, and at the same time remain in agreement with the physicality condition.

In [16] we showed that the $95 \%$ CL limit based on the $4.4 / \mathrm{fb}$ razor search is not affected by the change of the sign of parameter $\mu$. The same is true for the NUHM.

Limit combination procedure and results. In our approximate combination of the recent SUSY searches by CMS, we used all bins considered in the CMS $\alpha_{T} 11.7 / \mathrm{fb}, \sqrt{s}=$ $8 \mathrm{TeV}$ analysis [14], as well as the ones from the razor $4.4 / \mathrm{fb}, \sqrt{s}=7 \mathrm{TeV}$ [16]. Following the statistical approach of Modified Frequentist Confidence Levels [61] we assumed that the two searches are statistically independent (since they are based on different data sets) and we treated every bin as a statistically independent counting experiment. Then the combined likelihood is a product of the likelihoods for the two separate searches. The results of such an approximation are presented in figure 3(c), which shows a comparison of the $95 \%$ CL lines for the 5.8/fb ATLAS search at $\sqrt{s}=8 \mathrm{TeV}$ (dotted gray) and our combination of CMS results described above (solid red).

\subsection{Scanning tools and parameter ranges}

In this analysis we used the package BayesFITS which calls several external, publicly available tools: for sampling it uses MultiNest [62] with evidence tolerance factor set to 0.5, sampling efficiency equal to 0.8 , and number of live points equal to 4000 (CMSSM) or 10000 (NUHM).

Mass spectra were computed with SOFTSUSY v3.3.6 [63] and passed via SUSY Les Houches Accord format to SuperIso v3.3 [64] to calculate $\operatorname{BR}\left(\bar{B} \rightarrow X_{s} \gamma\right)$, 


\begin{tabular}{|l|l|l|l|}
\hline CMSSM parameter & Description & Prior Range & Prior Distribution \\
\hline$m_{0}$ & Universal scalar mass & $0.1,20$ & Log \\
$m_{1 / 2}$ & Universal gaugino mass & $0.1,10$ & Log \\
$A_{0}$ & Universal trilinear coupling & $-20,20$ & Linear \\
$\tan \beta$ & Ratio of Higgs vevs & 3,62 & Linear \\
$\operatorname{sgn} \mu$ & Sign of Higgs parameter & +1 or -1 & Fixed \\
\hline Nuisance & Description & Central value \pm std. dev. & Prior Distribution \\
\hline$M_{t}$ & Top quark pole mass & $173.5 \pm 1.0$ & Gaussian \\
$m_{b}\left(m_{b}\right)_{\mathrm{SM}}^{\overline{M S}}$ & Bottom quark mass & $4.18 \pm 0.03$ & Gaussian \\
$\alpha_{s}\left(M_{Z}\right)^{\overline{M S}}$ & Strong coupling & $0.1184 \pm 0.0007$ & Gaussian \\
$1 / \alpha_{\mathrm{em}}\left(M_{Z}\right)^{\overline{M S}}$ & Reciprocal of electromagnetic coupling & $127.916 \pm 0.015$ & Gaussian \\
\hline
\end{tabular}

Table 2. Priors for the parameters of the CMSSM and for the SM nuisance parameters used in our scans. Soft masses and $A_{0}$ are in TeV. Top quark pole mass and bottom quark mass are in GeV.

$\mathrm{BR}\left(B_{s} \rightarrow \mu^{+} \mu^{-}\right), \mathrm{BR}\left(B_{u} \rightarrow \tau \nu\right)$, and $\delta(g-2)_{\mu}^{\mathrm{SUSY}} \cdot \Delta M_{B_{s}}, \sin ^{2} \theta_{\text {eff }}$ and $M_{W}$ are calculated with FeynHiggs [65]. DM observables, such as the relic density and direct detection cross sections, are calculated with MicrOMEGAs 2.4.5 [66].

The prior ranges and metric adopted for scanning the CMSSM and nuisance parameters are given in table 2. We only scanned in log priors for the mass parameters, as it was proven in many previous studies [67] that flat priors in the CMSSM unduly favor the largescale regions of the parameter space (volume effect). Moreover, the correlation between $\mathrm{BR}\left(B_{s} \rightarrow \mu^{+} \mu^{-}\right)$and the $\mathrm{AF}$ region, which we expose in this study, becomes unobservable once large values of $m_{A}$ become favored by the scan.

Note that, compared to [16], we significantly extended the ranges of $m_{0}, m_{1 / 2}$ and $A_{0}$. We performed our scans for $\mu>0$ and $\mu<0$ separately. For negative $\mu$ we did not include the $(g-2)_{\mu}$ constraint, since its only effect would be to worsen the overall fit (see [16] for a detailed discussion of this issue), while the observable is very poorly fit anyway.

The prior ranges and metric for the NUHM parameters are given in table 3 . We performed several scans with different choices of ranges and priors. As we will explain in more detail in the next section, we selected the ranges that allowed us to most strongly see the possible correlation between $\operatorname{BR}\left(B_{s} \rightarrow \mu^{+} \mu^{-}\right)$and the AF region.

\section{Results}

In this section we will present our numerical results. We will first examine the impact of the current and the projected determination of $\mathrm{BR}\left(B_{s} \rightarrow \mu^{+} \mu^{-}\right)$on the different high probability regions of the parameter space of the CMSSM and will discuss ensuing implications for testing them. In particular, we will show that the AF region is likely to be basically fully excluded if the SM (or else current) value of $\mathrm{BR}\left(B_{s} \rightarrow \mu^{+} \mu^{-}\right)$is confirmed with high precision. Next we will demonstrate that future one-tonne detectors of dark matter scattering off nuclei will provide a crucial complementary way of cross-examining those regions and of potentially exploring the favored regions of the CMSSM over very wide ranges of parameters not accessible to direct LHC searches for new particles. Next 


\begin{tabular}{|l|l|l|l|}
\hline NUHM parameter & Description & Prior Range & Prior Distribution \\
\hline$m_{0}$ & Universal scalar mass & $0.1,4\left(0.1,20^{*}\right)$ & Log (Linear) \\
$m_{1 / 2}$ & Universal gaugino mass & $0.1,4(0.1,10)$ & Log (Linear) \\
$A_{0}$ & Universal trilinear coupling & $-7,7(-20,20)$ & Linear \\
$\tan \beta$ & Ratio of Higgs vevs & $15,35(3,62)$ & Linear \\
$\operatorname{sgn} \mu$ & Sign of Higgs parameter & +1 or -1 & Fixed \\
\hline$m_{H_{u}}$ & GUT-scale soft mass of $H_{u}$ & $0.1,4(0.1,20)$ & Linear \\
$m_{H_{d}}$ & GUT-scale soft mass of $H_{d}$ & $0.1,4(0.1,20)$ & Linear \\
\hline \multicolumn{5}{|l}{ Nuisance parameters like in the CMSSM } \\
\hline
\end{tabular}

${ }^{*}$ In parentheses we show the ranges for the scans giving the $1 \mathrm{TH}$ region, see section 4.2 .

Table 3. Priors for the parameters of the NUHM and for the SM nuisance parameters used in our scans. Soft masses and $A_{0}$ are in TeV. Top quark pole mass and bottom quark mass are in GeV.

we will apply a similar approach to the NUHM and show that the above conclusions in general will not hold. On the other hand, some positive measurements of DM signal will have the potential to basically rule out the CMSSM.

\subsection{The CMSSM}

In figures $4(\mathrm{a})$ and $4(\mathrm{~b})$ we plot $68 \%$ and $95 \%$ credibility regions of a two-dimensional (2D) marginalized posterior pdf (henceforth called posterior for brevity) in the $\left(m_{0}, m_{1 / 2}\right)$ plane and in the $\left(A_{0}, \tan \beta\right)$ plane, respectively, for $\mu>0$. In figures $4(\mathrm{c})$ and $4(\mathrm{~d})$ we show the same for $\mu<0$, but without $\delta(g-2)_{\mu}$, as mentioned earlier. The figures give an update and a significant extension of the results presented in our previous CMSSM analysis [16] by an inclusion of the new positive measurement of $\mathrm{BR}\left(B_{s} \rightarrow \mu^{+} \mu^{-}\right)$(instead of an upper limit) and by significantly extending mass parameter ranges; compare table 2 . In the ranges overlapping with those in the previous study $\left(0.1 \mathrm{TeV} \leq m_{0} \leq 4 \mathrm{TeV}, 0.1 \mathrm{TeV} \leq m_{1 / 2} \leq\right.$ $2 \mathrm{TeV},-7 \mathrm{TeV} \leq A_{0} \leq 7 \mathrm{TeV}$ and $\left.3 \leq \tan \beta \leq 62\right)$ the figures basically reproduce the same features, with the main three regions of high posterior favored primarily by the DM relic density and the Higgs mass, and also by the other constraints. First, the SC region shows up at small $m_{0}$ just above the LHC (CMS, and similarly for ATLAS) exclusion line. ${ }^{2}$ The posterior features a $68 \%$ credibility and the best-fit point is located there thanks to a very good fit to the Higgs mass, and a value of $\mathrm{BR}\left(B_{s} \rightarrow \mu^{+} \mu^{-}\right)$in agreement with the experiment (at not too large $\tan \beta$ ). Next, the AF region can be seen at the $2 \sigma$ credibility level for $1 \mathrm{TeV} \lesssim m_{0} \lesssim 4 \mathrm{TeV}$ and $1.2 \mathrm{TeV} \lesssim m_{1 / 2} \lesssim 2 \mathrm{TeV}$, although a much smaller $1 \sigma$ 'island' at smaller $m_{0}$ is also present. Finally, the $\mathrm{FP} / \mathrm{HB}$ region appears only as a $95 \%$ credibility island at $m_{0} \approx 4 \mathrm{TeV}$ due to the fact that it is more difficult there to produce the correct Higgs mass. (See [16] for a detailed discussion, and also [32] where we discussed in detail the CMSSM limit of the CNMSSM, and adopted the same updated values of experimental constraints as in this study.)

\footnotetext{
${ }^{2}$ The SC strip can be narrowed down by applying limits on long-lived charged particles to staus [25] but this will not significantly change the results presented here.
} 


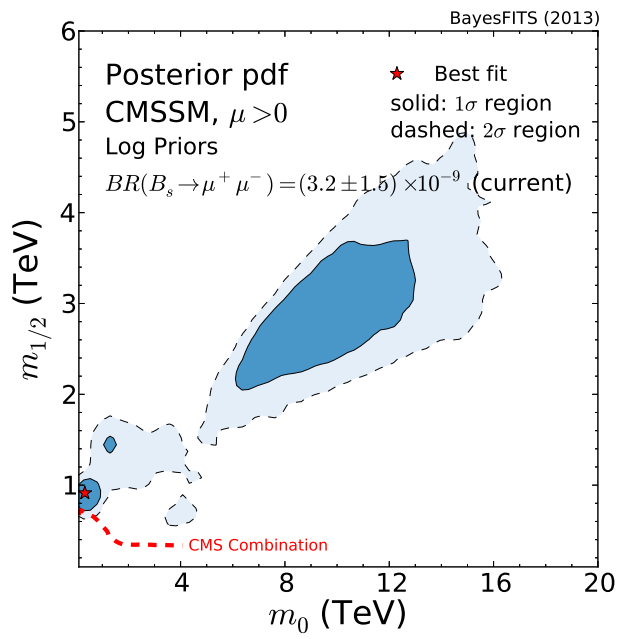

(a)

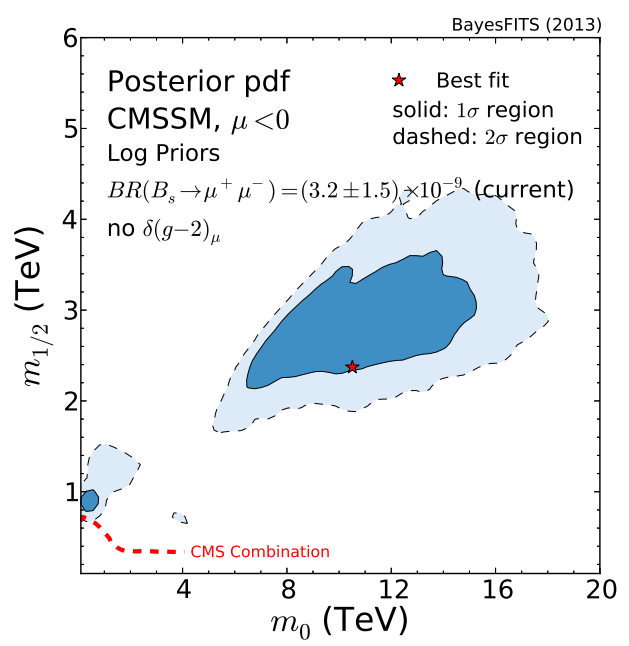

(c)

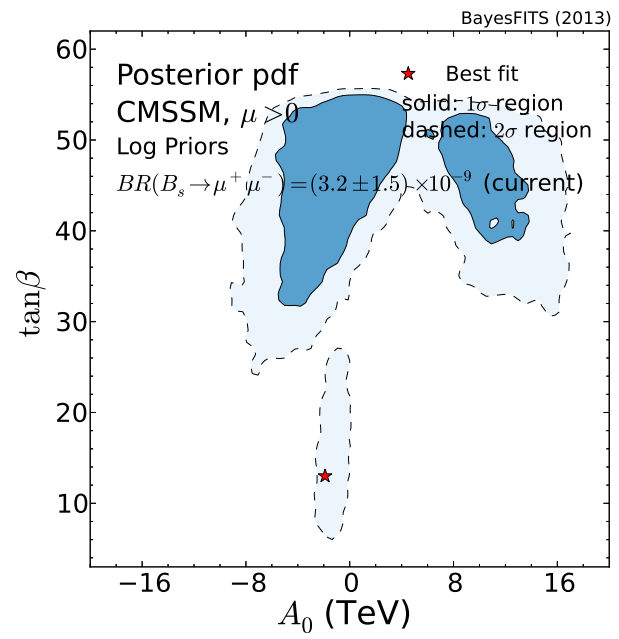

(b)

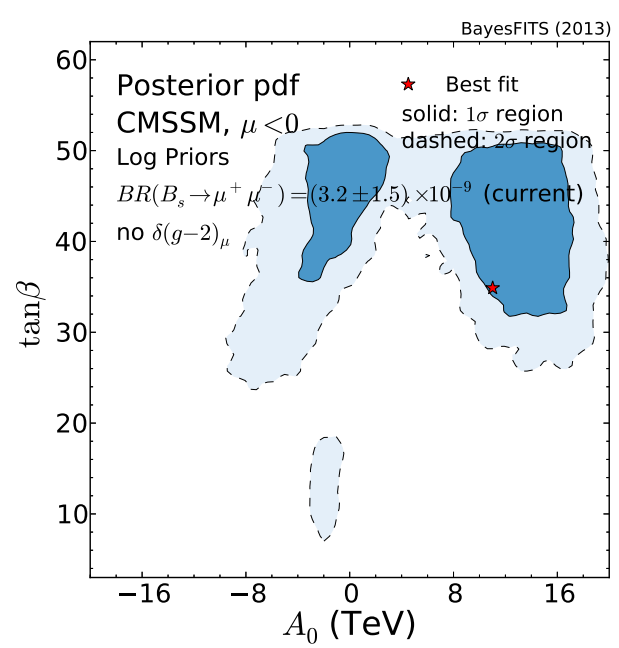

(d)

Figure 4. Marginalized 2D posterior pdf in (a) the $\left(m_{0}, m_{1 / 2}\right)$ plane of the CMSSM for $\mu>0$, (b) the $\left(A_{0}, \tan \beta\right)$ plane for $\mu>0$, (c) the $\left(m_{0}, m_{1 / 2}\right)$ plane for $\mu<0$, and (d) the $\left(A_{0}, \tan \beta\right)$ plane for $\mu<0$, constrained by the experiments listed in table 1, with the exclusion of $\delta(g-2)_{\mu}$ for $\mu<0$. The $68 \%$ credible regions are shown in dark blue, and the $95 \%$ credible regions in light blue. The dashed red line shows the CMS combined 95\% CL exclusion bound.

As a side remark, we note that in [16] the best-fit point was located in the AF region. ${ }^{3}$ With the new improved fit the best-fit point is now found in the SC region - this is due to the updated (somewhat increased) value of the top pole mass which made it easier to obtain a $126 \mathrm{GeV}$ Higgs mass in the SC region, also in the CNMSSM, as we discussed in detail in [32].

\footnotetext{
${ }^{3}$ It was also emphasized there that the location of the best-fit point in the CMSSM is very sensitive to exact values of input parameters, approximations used, etc.
} 
In the case of $\mu<0$ (but without $\delta(g-2)_{\mu}$ ) the AF region is much less prominent than for $\mu>0$, although still visible in figure 4 (c) at $95 \%$ credibility. Likewise the FP/HB region has shrunk considerably, while the SC remained fairly stable.

Going to larger $m_{0}$ and $m_{1 / 2}$, beyond those considered in [16], the main new feature in figures $4(\mathrm{a})$ and $4(\mathrm{c})$ is the appearance of a large $68 \%$ posterior region ranging from around 5 to $12 \mathrm{TeV}$ in $m_{0}$ [20] where the LSP is an almost purely higgsino-like neutralino with mass $m_{\chi} \approx \mu \simeq 1 \mathrm{TeV}$ (the $1 \mathrm{TH}$ region). The correct Higgs mass is also easily obtained there due to large $M_{\text {SUSY }}$ while all other constraints, including $\operatorname{BR}\left(B_{s} \rightarrow \mu^{+} \mu^{-}\right)$(except at large $\tan \beta)$, reproduce basically the SM value there. In fact, for $\mu<0$ with $\delta(g-2)_{\mu}$ dropped from the list of constraints, the best-fit point has now moved up to the $1 \mathrm{TH}$ region since no other constraint favors lower $M_{\mathrm{SUSY}}$.

Notice that figures 4(b) and 4(d) show that the parameters $A_{0}$ and $\tan \beta$ are now less constrained than in [16]. This is a consequence of extending the scanned ranges of $m_{0}$ and $m_{1 / 2}$ to much larger values. The large higgsino DM region corresponds to two large $68 \%$ credible regions, where $\tan \beta$ assumes values in the range $30-55$, and $A_{0}$ can take very large negative and positive values. The tree level value of $m_{H_{u}}^{2}$ is often positive, in which case electro-weak symmetry breaking (at the tree level) is not achieved. To overcome this, large and negative one-loop contributions to $m_{H_{u}}^{2}$, which are proportional to $\left|A_{t}\right| \tan \beta$, are needed. Therefore, at smaller $\tan \beta$ larger $\left|A_{t}\right|$ (and hence $\left|A_{0}\right|$ ) are favored - a tendency that becomes weaker as $\tan \beta$ grows. Hence, the posterior features a 'gap' for small $A_{0}$, which narrows down with increasing $\tan \beta\left(0 \lesssim A_{0} \lesssim 5 \mathrm{TeV}\right.$ implies $\left|A_{t}\right| \ll A_{0}$ for most choices of the other parameters). Values of $\tan \beta \lesssim 25$, on the other hand, are not favored in this region given the prior ranges considered in this study.

At this point one can raise the question whether the $95 \%$ credibility upper bound to the $1 \mathrm{TH}$ region $\left(m_{0} \approx 16 \mathrm{TeV}, m_{1 / 2} \approx 5 \mathrm{TeV}\right)$ is due to the physical impact of the constraints considered here, or is rather a feature of our choice of priors and parameter ranges. We will explain this in what follows.

In the regime of such large masses, SUSY contributions to all electroweak and flavor observables become very small, so that the only constraints that can affect the favored parameter space are the relic density and the Higgs mass. It has being long known that the loop corrections to the Higgs mass increase logarithmically with increasing $M_{\text {SUSY }}$ so that, in principle, the measured value of the Higgs mass can place an upper limit on $m_{0}$, $m_{1 / 2}$. To exemplify this feature we show in figure 5 (a) the marginalized posterior pdf in the $\left(m_{0}, m_{1 / 2}\right)$ plane for the parameter ranges considered in this analysis, in the case where all other constraints with the exception of the Higgs mass and SUSY limits from the LHC are turn off. One can see that the $68 \%$ credibility region does not extend beyond $m_{0} \simeq 14 \mathrm{TeV}$ and $m_{1 / 2} \simeq 6 \mathrm{TeV}$. In figure $5(\mathrm{~b})$ we show the marginalized $1 \mathrm{D}$ posterior pdf for the Higgs mass in this case (solid blue line), to confirm that $m_{h}$ can reproduce the experimental value very well with these parameter ranges. Moreover, the nearly Gaussian shape of the 1D pdf implies basically no tension with the CMS lower limit in the $\left(m_{0}, m_{1 / 2}\right)$ plane, as the $68 \%$ credibility region favored by the Higgs mass favors multi-TeV scale for both CMSSM parameters. In other words, the Higgs mass of around $126 \mathrm{GeV}$ typically implies $M_{\text {SUSY }}$ in the range of a few to several $\mathrm{TeV}$. 


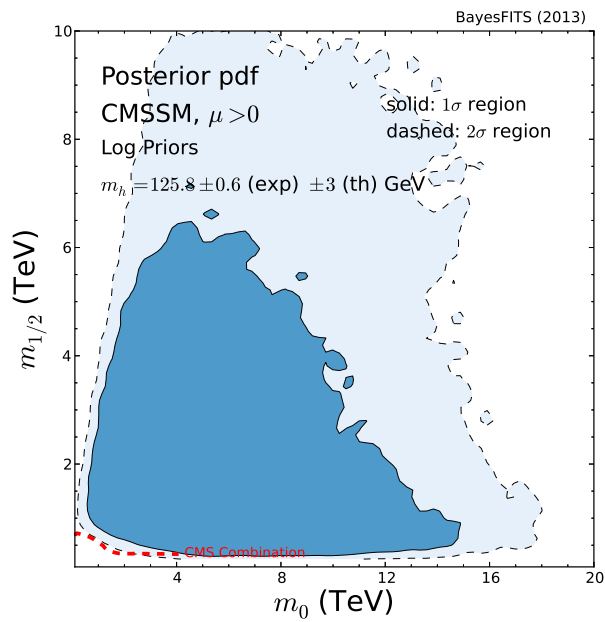

(a)

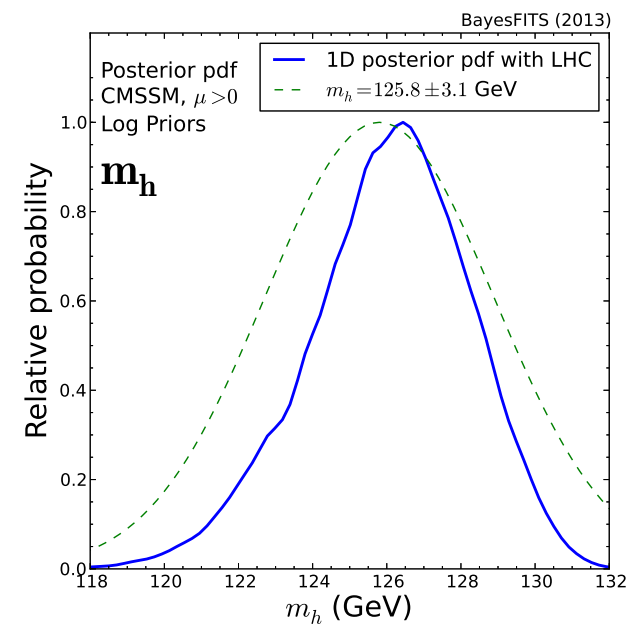

(b)

Figure 5. (a) Marginalized 2D posterior pdf in the $\left(m_{0}, m_{1 / 2}\right)$ plane of the CMSSM constrained only by the Higgs mass and the LHC SUSY searches. The $68 \%$ credible regions are shown in dark blue, and the $95 \%$ credible regions in light blue. The dashed red line shows the CMS combined 95\% CL exclusion bound. (b) Marginalized 1D posterior pdf for $m_{h}$ (solid blue line) under the same assumptions as in (a). The dashed green line shows the Higgs mass likelihood, with experimental and theoretical uncertainties added in quarature.

However, the upper bound on $m_{0}$ and $m_{1 / 2}$ shown in figure $5(\mathrm{a})$ does depend on the assumed parameter range and on the prior distribution. We have checked that, by extending the parameter space to $m_{0}, m_{1 / 2}=50 \mathrm{TeV}$ and $A_{0}= \pm 50 \mathrm{TeV}$, the $68 \%$ and $95 \%$ credibility bounds in the $\left(m_{0}, m_{1 / 2}\right)$ plane extend by approximately $50 \%$ in both directions when maintaining log priors, and by $50 \%$ in $m_{0}$ and a factor of two in $m_{1 / 2}$ when switching to flat priors. Furthermore, it was recently shown in a detailed study [68] that for values of $\tan \beta$ lower than the ones considered in this study $(1 \leq \tan \beta<3$, disfavored by the relic density constraint) there is vitually no bound on $M_{\text {SUSY }}$ due to the Higgs mass, up to GUT scale.

On the other hand, the relic density does impose a much stronger bound on the favored parameter space. In the high-mass region, the tree-level $\mu$ parameter and the one-loop tadpole corrections to its value can both significantly exceed the $1 \mathrm{TeV}$ scale. Since the relic density constraint in the $1 \mathrm{TH}$ region requires $\mu \sim 1 \mathrm{TeV}$, as explained above, treelevel and one-loop contributions should cancel each other with very high accuracy, which requires precise tuning of the model parameters. This also affects the stability of the solutions provided by the spectrum generators (see [69] for a detailed discussion). In fact, for $m_{0}>20 \mathrm{TeV}$ it becomes very difficult to generate spectra with $\mu \sim 1 \mathrm{TeV}$, and it becomes virtually impossible for $m_{0}>40 \mathrm{TeV}$. This causes an upper bound on the highprobability higgsino regions shown in figures $4(\mathrm{a})$ and $4(\mathrm{c})$.

Notice that, given the very strong constraints from the relic abundance, the upper bound on the $1 \mathrm{TH}$ region is basically range and prior independent. We checked this with 


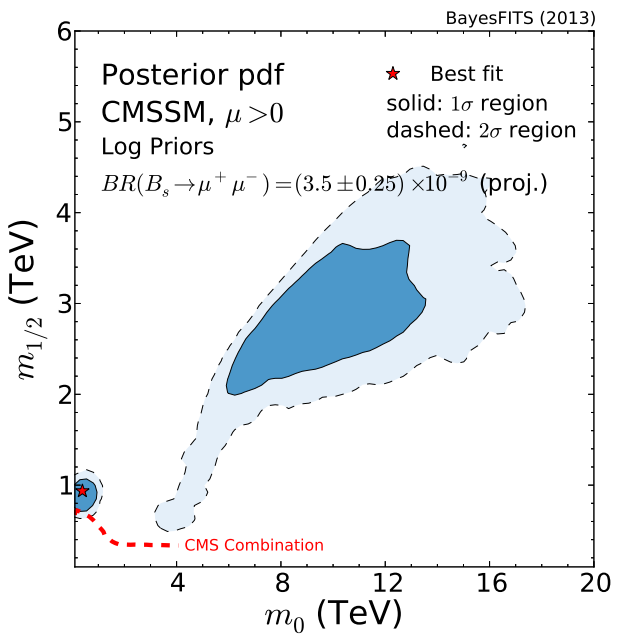

(a)

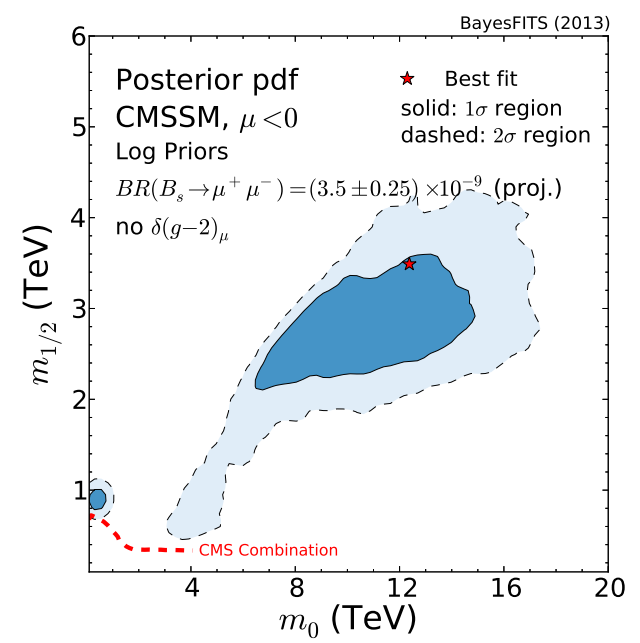

(b)

Figure 6. Marginalized 2D posterior pdf in the $\left(m_{0}, m_{1 / 2}\right)$ plane of the CMSSM constrained by the experiments listed in table 1 with projected uncertainties for $\operatorname{BR}\left(B_{s} \rightarrow \mu^{+} \mu^{-}\right)$. (a) $\mu>0$, (b) $\mu<0$. The $68 \%$ credible regions are shown in dark blue, and the $95 \%$ credible regions in light blue. The dashed red line shows the CMS combined 95\% CL exclusion bound.

a supplementary scan, with all constraints included, in which we extended the parameter ranges up to $50 \mathrm{TeV}$ for $m_{0}$ and up to $20 \mathrm{TeV}$ for $m_{1 / 2}$, with log and linear priors. The upper bound to the $1 \mathrm{TH}$ region in the $\left(m_{0}, m_{1 / 2}\right)$ plane remained virtually unchanged.

In figures $6(\mathrm{a})$ and $6(\mathrm{~b})$ we show the marginalized $2 \mathrm{D}$ posterior pdf in the $\left(m_{0}, m_{1 / 2}\right)$ plane for $\mu>0$ and $\mu<0$, respectively, for the scans where we adopted projected future theoretical and experimental uncertainties (added in quadrature) for $\operatorname{BR}\left(B_{s} \rightarrow \mu^{+} \mu^{-}\right)$, i.e., $\mathrm{BR}\left(B_{s} \rightarrow \mu^{+} \mu^{-}\right)$proj $=(3.5 \pm 0.25) \times 10^{-9}$. The AF region does not appear in the high posterior anymore, even at $95 \%$ credibility, while the other regions basically do not change. This was to be expected in light of the discussion presented in section 2 . The argument is valid for both signs of $\mu$ : when $\mu>0(\mu<0) \mathrm{BR}\left(B_{s} \rightarrow \mu^{+} \mu^{-}\right)$in the AF region assumes much larger (smaller) values than the ones favored by the projected uncertainties, as shown qualitatively in figure $1(\mathrm{~d})$. The location of the best-fit point is different for $\mu<0$ since, like in figure $4(\mathrm{c})$, the constraint from $\delta(g-2)_{\mu}$ has not been included in the likelihood function.

The results shown in figure 6 are quite insensitive to the projected uncertainties assumed for $\operatorname{BR}\left(B_{s} \rightarrow \mu^{+} \mu^{-}\right)$. The shape of the posterior pdf does not change even if they are doubled, which we have checked numerically. We also point out that the situation will not be different if in the future the uncertainties are narrowed around the currently measured central value $\left(3.2 \times 10^{-9}\right)$ instead of the SM value. We also checked this numerically, finding no significant difference in the resulting posterior.

To highlight the fact that in the CMSSM a more precise determination of $\mathrm{BR}\left(B_{s} \rightarrow \mu^{+} \mu^{-}\right)$can lead to an almost complete exclusion of the AF region, we show 


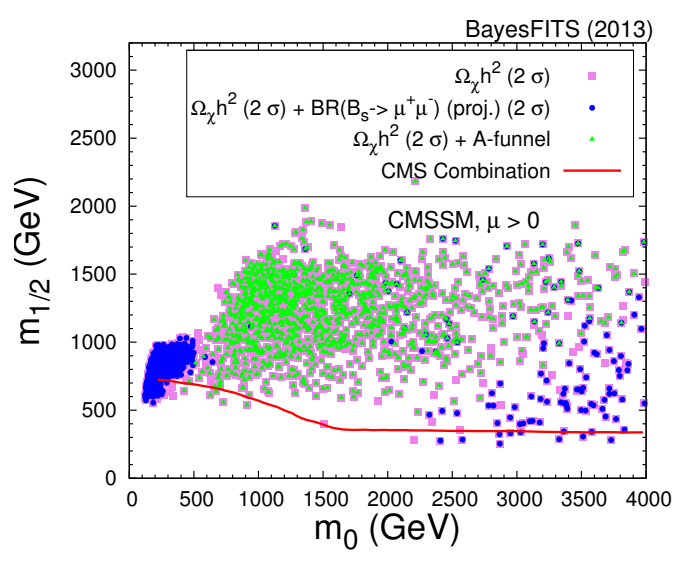

(a)

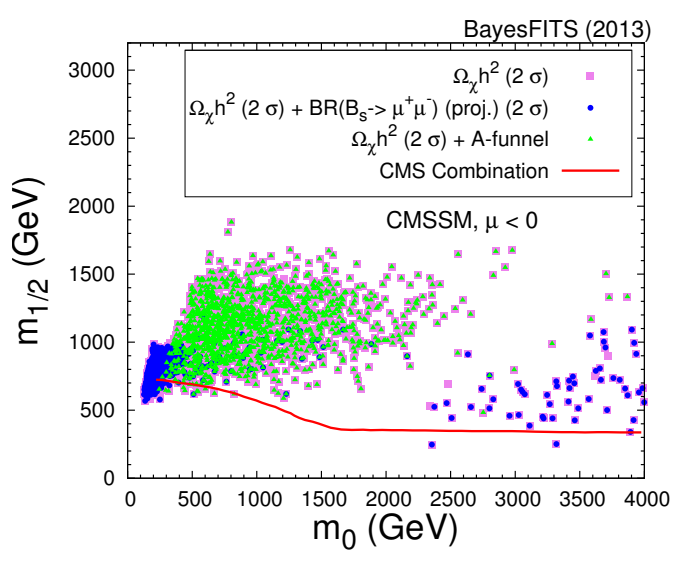

(b)

Figure 7. Scatter plot of the points in the $\left(m_{0}, m_{1 / 2}\right)$ plane of the CMSSM for (a) $\mu>0$, and (b) $\mu<0$, satisfying $\Omega_{\chi} h^{2}$ at $2 \sigma$ (pink squares), $\Omega_{\chi} h^{2}+\mathrm{BR}\left(B_{s} \rightarrow \mu^{+} \mu^{-}\right)$at $2 \sigma$ (blue circles), and $\Omega_{\chi} h^{2}$ at $2 \sigma$ and $\left|m_{A}-2 m_{\chi}\right|<100 \mathrm{GeV}$ (green triangles).

in figure 7 scatter plots of the points of our chains restricted to the low-mass regions. In figure 7 (a) $\mu>0$, whereas in figure 7 (b) $\mu<0$. Pink squares mark the points for which the relic density constraint is satisfied at $2 \sigma$ (theoretical + experimental errors added in quadrature); blue circles represent the subset of these points for which $\mathrm{BR}\left(B_{s} \rightarrow \mu^{+} \mu^{-}\right)$ is satisfied at projected $2 \sigma$ (with total $\sigma=0.25 \times 10^{-9}$ ); green triangles mark the subset of these points that belong to the AF region $\left(\left|m_{A}-2 m_{\chi}\right|<100 \mathrm{GeV}\right)$. One can see a good spacial separation between the blue and green points, which is a reflection of the tension of the $\mathrm{AF}$ region with the $\mathrm{BR}\left(B_{s} \rightarrow \mu^{+} \mu^{-}\right)$constraint.

The mass scales typical for the AF region are so high that most of it will remain beyond direct reach of the LHC. (For example, with $300 / \mathrm{fb}$ at $14 \mathrm{TeV}$, CMS will probe $m_{1 / 2} \lesssim 1.3-1.4 \mathrm{TeV}$ at $m_{0} \lesssim 1 \mathrm{TeV}[70]$.) Likewise, the $\mathrm{FP} / \mathrm{HB}$ region will also be only partially probed at the $\mathrm{LHC}$, while the $1 \mathrm{TH}$ region will remain completely beyond direct collider reach. As we have demonstrated, the projected precision in the determination of $\mathrm{BR}\left(B_{s} \rightarrow \mu^{+} \mu^{-}\right)$will have the power to potentially rule out the $\mathrm{AF}$ region, but not the other ones.

Fortunately, an expected ultimate sensitivity of DM searches in deep underground detectors will provide a crucial complementary, and partly overlapping, venue of testing all the high posterior probability regions of the CMSSM. In figures 8(a) and 8(b) we show a 2D posterior in the $\left(m_{\chi}, \sigma_{p}^{\mathrm{SI}}\right)$ plane for $\mu>0$ and $\mu<0$, respectively. Starting from figure 8 (a), we can clearly identify the four high posterior probability regions, each with a characteristic LSP mass range and $\sigma_{p}^{\mathrm{SI}}$. The SC region (appearing only at $95 \%$ credibility but featuring the best-fit point) corresponds to fairly low $m_{\chi}(\lesssim 450 \mathrm{GeV})$ and typically the lowest $\sigma_{p}^{\mathrm{SI}}$. Next to it, with somewhat larger $m_{\chi}$ and $\sigma_{p}^{\text {SI }}$ lies the AF region (also at $2 \sigma$ credibility), which in turn is very well separated from the big $68 \%$ credibility region of $\sim 1 \mathrm{TeV}$ higgsino LSP. Finally, the spin-independent cross section in the FP/HB region featuring a mixed 


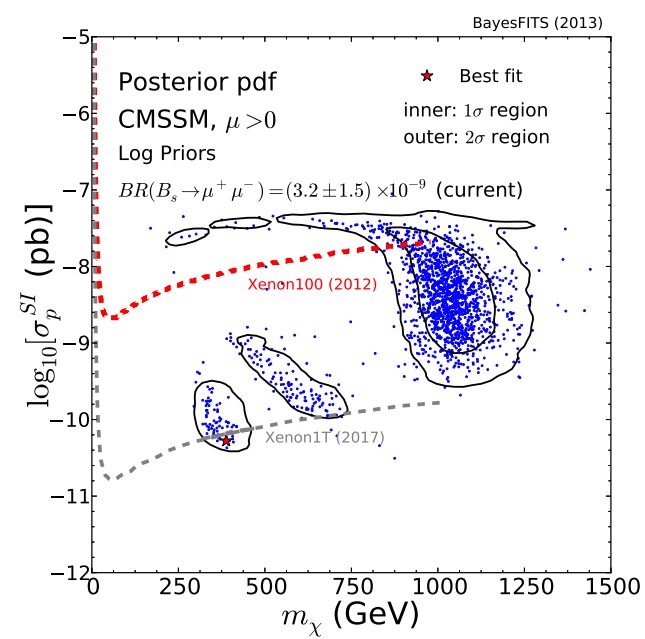

(a)

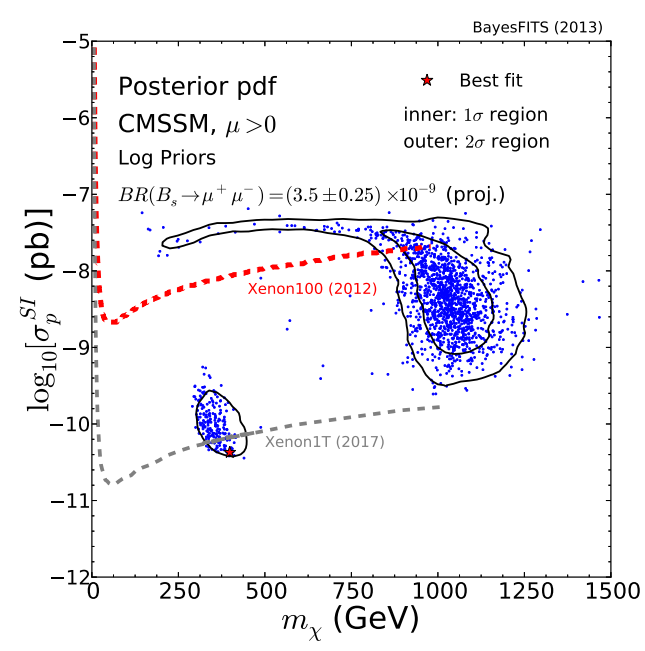

(c)

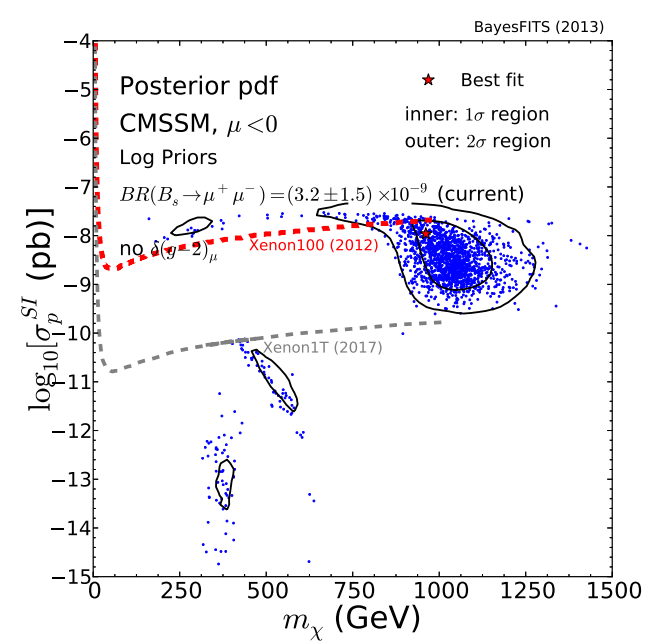

(b)

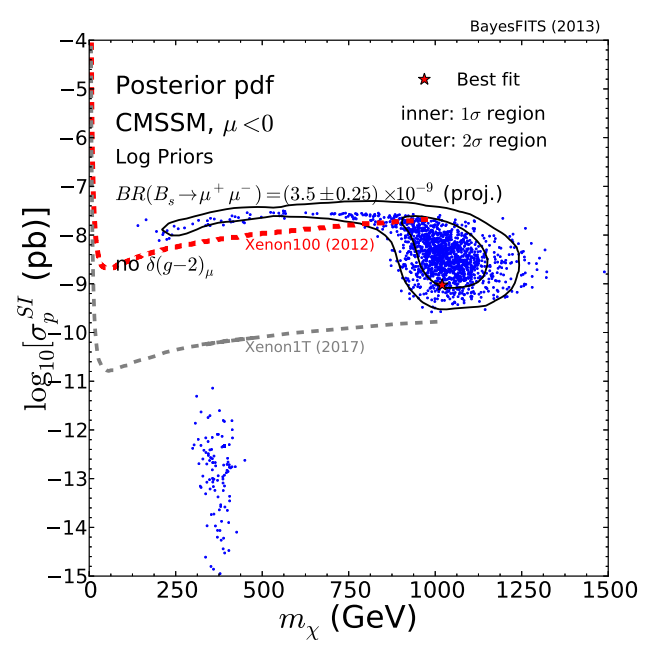

(d)

Figure 8. Marginalized 2D posterior pdf in the $\left(m_{\chi}, \sigma_{p}^{\mathrm{SI}}\right)$ plane for the CMSSM constrained by the experiments listed in table 1. (a) $\mu>0$, current uncertainties in $\operatorname{BR}\left(B_{s} \rightarrow \mu^{+} \mu^{-}\right)$, (b) $\mu<0$, current uncertainties in $\operatorname{BR}\left(B_{s} \rightarrow \mu^{+} \mu^{-}\right)$, (c) $\mu>0$, projected uncertainties in $\operatorname{BR}\left(B_{s} \rightarrow \mu^{+} \mu^{-}\right)$, and (d) $\mu<0$, projected uncertainties in $\mathrm{BR}\left(B_{s} \rightarrow \mu^{+} \mu^{-}\right)$. The dashed red line show the $90 \% \mathrm{CL}$ exclusion bound by XENON100 (not included in the likelihood), and the dashed gray line the projected sensitivity for XENON-1T. A distribution of samples uniformly selected from our nested sampling chain is superimposed.

bino-higgsino neutralino (a horizontal branch at $\sigma_{p}^{\mathrm{SI}} \simeq 3 \times 10^{-8} \mathrm{pb}$ ) already shows tension with the current 90\% CL upper bound from XENON100 [30]. However, as demonstrated in [47] and mentioned above, this region is probably not yet firmly excluded due to large theoretical and astrophysical uncertainties. The remaining three regions are currently below the XENON100 exclusion line but will be almost entirely probed by future onetonne detectors, as a projected sensitivity line for XENON-1T [71] indicates. Note that, in 


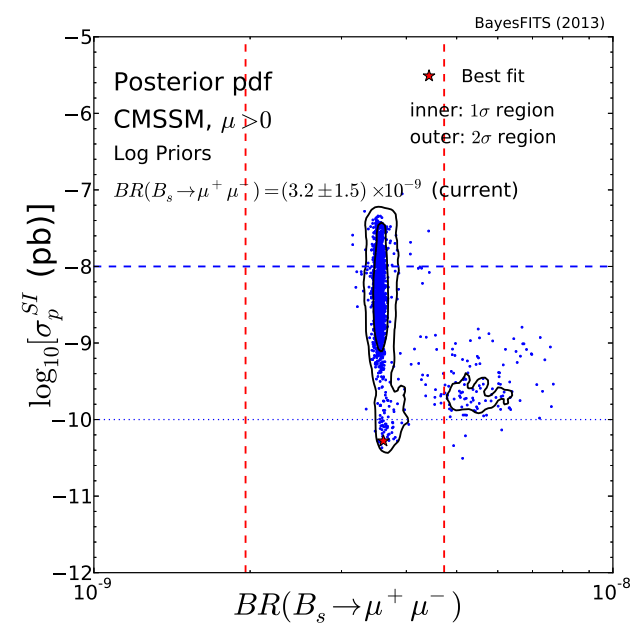

(a)

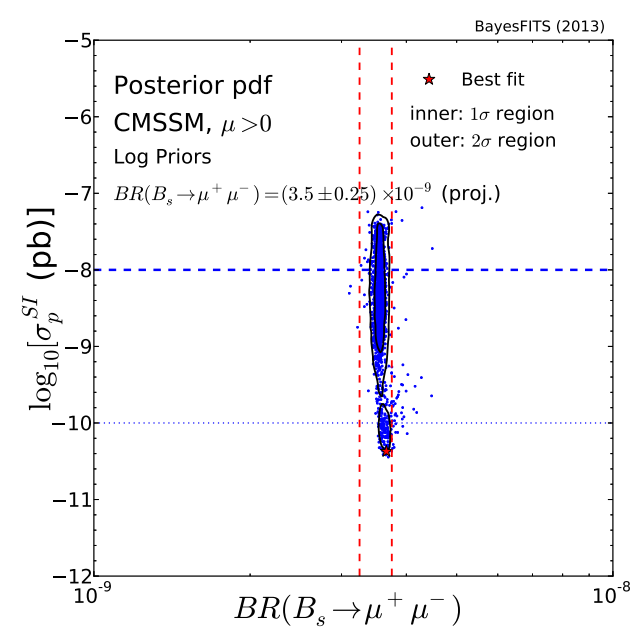

(c)

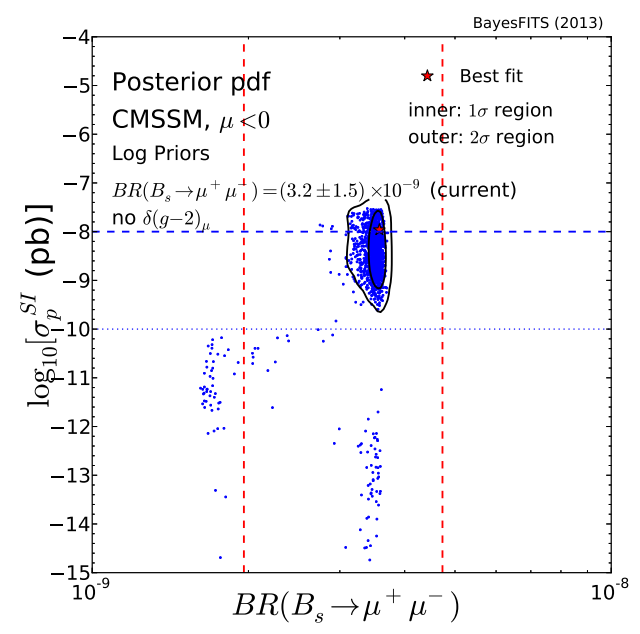

(b)

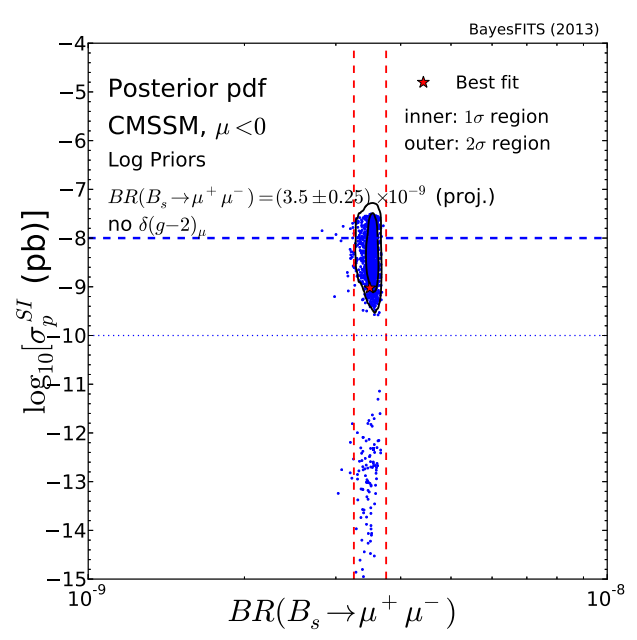

(d)

Figure 9. Marginalized $2 \mathrm{D}$ posterior pdf in the $\left(\mathrm{BR}\left(B_{s} \rightarrow \mu^{+} \mu^{-}\right), \sigma_{p}^{\mathrm{SI}}\right)$ plane for the CMSSM constrained by the experiments listed in table 1. (a) $\mu>0$, current uncertainties in $\operatorname{BR}\left(B_{s} \rightarrow \mu^{+} \mu^{-}\right)$, (b) $\mu<0$, current uncertainties in $\operatorname{BR}\left(B_{s} \rightarrow \mu^{+} \mu^{-}\right)$, (c) $\mu>0$, projected uncertainties in $\mathrm{BR}\left(B_{s} \rightarrow \mu^{+} \mu^{-}\right)$, and (d) $\mu<0$, projected uncertainties in $\operatorname{BR}\left(B_{s} \rightarrow \mu^{+} \mu^{-}\right)$. The dashed red vertical lines show the current [(a) and (b)] and projected [(c) and (d)] uncertainties on $\operatorname{BR}\left(B_{s} \rightarrow \mu^{+} \mu^{-}\right)$at $1 \sigma$. A distribution of samples uniformly selected from our nested sampling chain is superimposed. The dashed horizontal line shows the minimum $90 \%$ CL bound by XENON100, and the dotted horizontal line the corresponding projected sensitivity for XENON1T.

the absence in the likelihood function of any constraint to favor the SC or AF regions, the broad ranges of the CMSSM input parameter assumed for our scans the make posterior strongly favor the $1 \mathrm{TH}$ region which presents the vast majority of points (the volume effect) even with the log prior on $m_{0}$ and $m_{1 / 2}$, although at $95 \%$ of total posterior probability, the other regions are also present. We also note that in random scans one can find points 
with reasonably good $\chi^{2}\left(\delta \chi^{2} \leq 12\right)$ lying beyond those favored regions. We illustrate this by superimposing on the posterior a distribution of samples uniformly selected from our nested sampling chain (blue dots).

For comparison with the situation at present, figures $8(\mathrm{c})$ and $8(\mathrm{~d})$ show the same posterior in the case where the future projected uncertainties on $\operatorname{BR}\left(B_{s} \rightarrow \mu^{+} \mu^{-}\right)$are assumed; in other words $\mathrm{BR}\left(B_{s} \rightarrow \mu^{+} \mu^{-}\right)$is assumed to be basically reproducing the SM value. For $\mu>0$ the $\mathrm{AF}$ region is now gone and there remain essentially two testable regions: the $1 \mathrm{TH}$ region, which should be basically fully reachable by future DM searches only, and the SC region, testable also in part by direct searches at the LHC. Furthermore, they are so widely separated in the plane that a detection of a DM signal, even with poor initial determination of both $m_{\chi}$ and $\sigma_{p}^{\text {SI }}$, would have the power to discriminate between them. Furthermore, for $\mu<0$ the CMSSM predicts that only the higgsino region will be reachable by one-tonne detectors, while in the SC region a well known cancellation of two terms reduces $\sigma_{p}^{\text {SI }}$ to hopelessly low values. This actually gives one a chance, even if somewhat indirect one, to additionally determine the sign of $\mu$ since any DM measurement indicative of the SC region would most likely favor the positive sign of $\mu$.

Some of the points made above are recast in a somewhat different way in figure 9 where we plot $2 \mathrm{D}$ posterior regions in the $\left(\mathrm{BR}\left(B_{s} \rightarrow \mu^{+} \mu^{-}\right), \sigma_{p}^{\mathrm{SI}}\right)$ plane assuming the current (upper panels) and projected (lower panels) determination of $\operatorname{BR}\left(B_{s} \rightarrow \mu^{+} \mu^{-}\right)$, as indicated with vertical bars showing the combined (theory + experimental) errors. At present the AF region lies (for both signs of $\mu$ ) mostly (at $95 \%$ credibility level) beyond the current $1 \sigma$ experimental lines, and clearly not yet firmly excluded. However, after the projected uncertainties on $\mathrm{BR}\left(B_{s} \rightarrow \mu^{+} \mu^{-}\right)$are assumed, as shown in figures $9(\mathrm{c})$ and $9(\mathrm{~d})$, only the two testable regions mentioned above survive, the $1 \mathrm{TH}$ region corresponding to larger $\sigma_{p}^{\mathrm{SI}}$ and the SC region at the borderline of XENON-1T reach $(\mu>0)$ or below it $(\mu<0)$.

\subsection{The NUHM}

We have demonstrated above that projected but realistic sensitivities of $\operatorname{BR}\left(B_{s} \rightarrow \mu^{+} \mu^{-}\right)$ will have the discriminating power to basically rule out the AF region in the CMSSM. Furthermore, future one-tonne detectors of DM will reach down to values of $\sigma_{p}^{\mathrm{SI}}$ such that either a signal in one of the two remaining high posterior probability regions is detected, or the CMSSM will basically be ruled out over very wide ranges of its parameters (with the exception of the $\mathrm{SC}$ region at negative $\mu$ ), thus reaching far above the direct sparticle mass reach at the LHC. On the other hand, by detecting a DM signal at low $m_{\chi}$ the sign of $\mu$ could potentially also be determined.

The question arises whether such rather strong statements extend beyond the CMSSM. Unfortunately, it is easy to see that this is not the case already in the NUHM, which is one of the simplest extensions of the CMSSM. As mentioned above, in the NUHM, one can choose $m_{A}$ and $\mu$ as the additional two free parameters; see eqs. (2.9)-(2.10). These are precisely the quantities that played the crucial role in the CMSSM where they were, however, tightly constrained. On the other hand, we will show that in the NUHM one predicts some signatures for DM searches in one-tonne detectors that are absent in the 


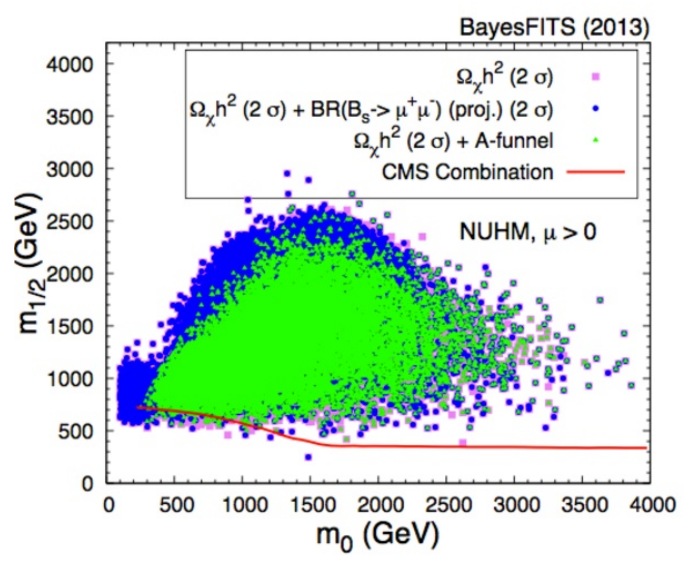

(a)

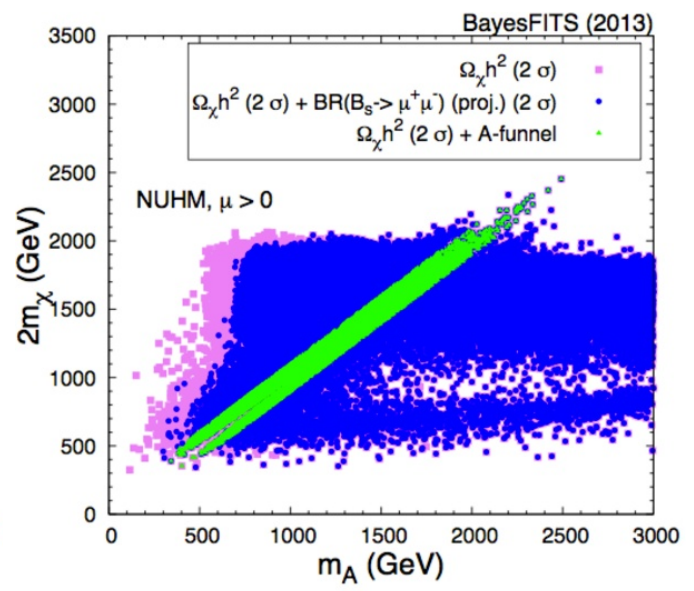

(b)

Figure 10. Scatter plot of the points in the (a) $\left(m_{0}, m_{1 / 2}\right)$ plane and (b) $\left(m_{A}, 2 m_{\chi}\right)$ plane of the NUHM for $\mu>0$ that satisfy $\Omega_{\chi} h^{2}$ at $2 \sigma$ (pink squares), $\Omega_{\chi} h^{2}+\mathrm{BR}\left(B_{s} \rightarrow \mu^{+} \mu^{-}\right)$at $2 \sigma$ (blue circles), and $\Omega_{\chi} h^{2}$ at $2 \sigma$ and $\left|m_{A}-2 m_{\chi}\right|<100 \mathrm{GeV}$ (green triangles).

CMSSM - this could provide the way for ruling out the latter model over multi-TeV ranges of mass parameters.

Since the enlarged parameter space of the NUHM, with much more freedom in the Higgs sector, allows a very good fit to almost all observables (except invariably $\delta(g-2)_{\mu}$ ), it is very time consuming to perform a global Bayesian scan as above for the CMSSM. Additionally one has to worry about much stronger prior dependence and volume effect [31]. However, since our goal in this paper is to examine the impact of future $\operatorname{BR}\left(B_{s} \rightarrow \mu^{+} \mu^{-}\right)$ and direct DM search sensitivities, a scan over a much more limited range of priors, given in table 3 , is sufficient to provide a counter-example to the conclusions drawn above in the CMSSM. Furthermore, we will not need to draw Bayesian high posterior regions to make our point.

In figure 10(a) we present, for $\mu>0$, the distribution of points (pink squares) in the $\left(m_{0}, m_{1 / 2}\right)$ plane for which the value of the relic density does not exceed the central value by more than $2 \sigma$. (Since the relic abundance is a strong constraint with a very small uncertainty, the distribution of points determines $95 \%$ credibility regions of the $2 \mathrm{D}$ pdf to very good accuracy but we don't show them here.) In green we show the subset of these points for which the correct relic density is obtained through neutralino annihilation via the $A$-resonance. These points constitute the AF region of the NUHM. We also show in blue the subset of the pink points that will additionally satisfy the constraint on $\operatorname{BR}\left(B_{s} \rightarrow \mu^{+} \mu^{-}\right)$ within the projected $2 \sigma$ error. In figure $10(\mathrm{~b})$ we show the same sets of points in the $\left(m_{A}\right.$, $2 m_{\chi}$ ) plane, to highlight the features of the AF region. A very similar pattern emerges for $\mu<0$, hence we do not show it here.

By comparing these figures with figure 7 , one can see that, in contrast to the CMSSM, in the NUHM a precise determination of $\mathrm{BR}\left(B_{s} \rightarrow \mu^{+} \mu^{-}\right)$will have no real discriminating power over the regions of the $\left(m_{0}, m_{1 / 2}\right)$ plane, as the points do not show spacial sepa- 


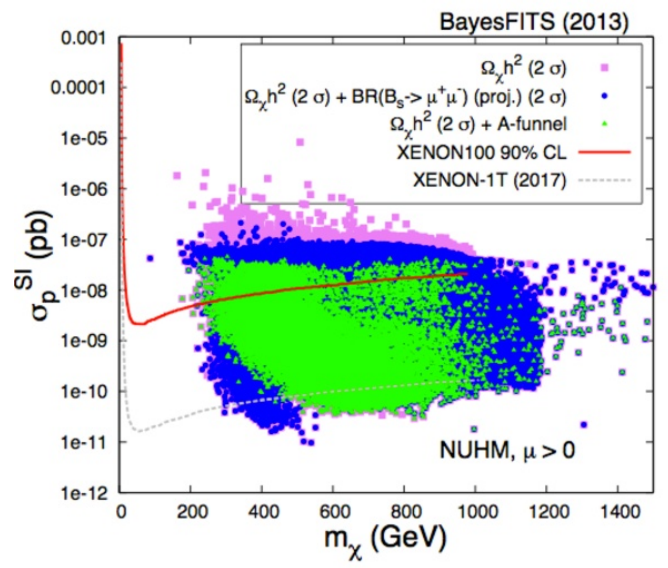

(a)

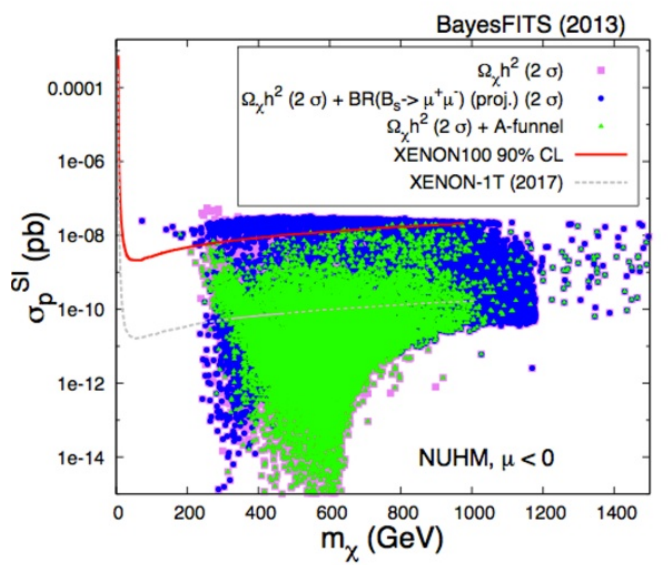

(b)

Figure 11. Scatter plot of the points in the $\left(m_{\chi}, \sigma_{p}^{\mathrm{SI}}\right)$ plane of the NUHM for (a) $\mu>0$, and (b) $\mu<0$, that satisfy $\Omega_{\chi} h^{2}$ at $2 \sigma$ (pink squares), $\Omega_{\chi} h^{2}+\mathrm{BR}\left(B_{s} \rightarrow \mu^{+} \mu^{-}\right)$at $2 \sigma$ (blue circles), and $\Omega_{\chi} h^{2}$ at $2 \sigma$ and $\left|m_{A}-2 m_{\chi}\right|<100 \mathrm{GeV}$ (green triangles).

ration. In other words, the $\mathrm{AF}$ region will remain prominently allowed even if a future determination of $\mathrm{BR}\left(B_{s} \rightarrow \mu^{+} \mu^{-}\right)$will narrow it down to basically the $\mathrm{SM}$ value.

The same, unfortunately, is true when it comes to future direct detection of DM. In figures $11(\mathrm{a})$ and $11(\mathrm{~b})$ we show the same points in the $\left(m_{\chi}, \sigma_{p}^{\mathrm{SI}}\right)$ plane for $\mu>0$ and $\mu<0$, respectively. One can see that the green points featuring the AF region with good relic density and SM-like $\mathrm{BR}\left(B_{s} \rightarrow \mu^{+} \mu^{-}\right)$cover very wide ranges of both $m_{\chi}$ and $\sigma_{p}^{\mathrm{SI}}$, extending from the SC region to the $1 \mathrm{TH}$ region, which is also present in the NUHM [31]; for a recent update see [23]. One needs to remember that the limited prior ranges used here for the NUHM (see table 3 ) do not fully reproduce the large $1 \mathrm{TH}$ region at $m_{\chi} \simeq 1 \mathrm{TeV}$. Nevertheless, we added to the plots the points of the $1 \mathrm{TH}$ region that were obtained with extra scans featuring linear priors in all mass parameters, and much broader ranges (also given in table 3). This is allowed, as long as we do not draw any statistical conclusion from the combination of these chains. One can see from figure 11 that it will be much more challenging to discriminate among the three high probability regions: a DM signal detected at smaller $m_{\chi}$ could be indicative of either the $\mathrm{SC}$ or the $\mathrm{AF}$ region while the same at $m_{\chi}$ close to $1 \mathrm{TeV}$ could instead imply either the AF or the $1 \mathrm{TH}$ region. Furthermore, for negative $\mu$, in a large number of cases with good dark matter relic density and SM-like $\mathrm{BR}\left(B_{s} \rightarrow \mu^{+} \mu^{-}\right)$(and correct Higgs mass, etc) accidental cancellations produce $\sigma_{p}^{\mathrm{SI}}$ well below the reach of even one-tonne detectors.

On the positive side, there is one class of DM signal measurements that could potentially allow one to basically rule out the CMSSM over a whole reasonable range of parameters. The NUHM prominently features a wide region of roughly $500 \mathrm{GeV} \lesssim m_{\chi} \lesssim 800 \mathrm{GeV}$ and $\sigma_{p}^{\text {SI }}$ often within the reach of one-tonne detectors which is absent in the CMSSM (except for a handful of cases with relatively poor $\chi^{2}$; compare figure $8(\mathrm{c})$ ). A detection of a signal in future DM searches indicative of this mass range would then provide a strong argument against the CMSSM. 


\section{Summary and conclusions}

In this paper we have examined the implications from the current and the projected but realistic sensitivities of both $\mathrm{BR}\left(B_{s} \rightarrow \mu^{+} \mu^{-}\right)$at the LHC and $\sigma_{p}^{\mathrm{SI}}$ in direct DM searches on the CMSSM and the NUHM. Within the CMSSM we performed an updated global Bayesian analysis of the CMSSM, with particular focus on the impact of the recent measurement of $\mathrm{BR}\left(B_{s} \rightarrow \mu^{+} \mu^{-}\right)$at $\mathrm{LHCb}$. We further extended the parameter ranges with respect to our previous analysis of the model, and we updated the limits from CMS direct SUSY searches through our likelihood map procedure, obtained by simulating the SUSY signal and the detector efficiencies. We showed that the same lower bounds apply to the NUHM as well. We confirmed that, in the CMSSM, in addition to the previously identified high posterior probability regions of the $\left(m_{0}, m_{1 / 2}\right)$ plane favored by the global constraints, at previously unexplored large CMSSM mass scales a prominent $68 \%$ credibility region appears, where the LSP is a nearly pure higgsino with mass of about $1 \mathrm{TeV}$.

We highlighted a correlation between $\operatorname{BR}\left(B_{s} \rightarrow \mu^{+} \mu^{-}\right)$and the $A$-funnel region of the CMSSM as the above branching ratio and the annihilation cross section in the AF region both primarily depend on the same parameters: $m_{A}$ and $\tan \beta$. In this regard, we showed that the AF region of the CMSSM is at present slightly disfavored (95\% credibility of the posterior pdf) by the first $\mathrm{BR}\left(B_{s} \rightarrow \mu^{+} \mu^{-}\right)$measurement, although far from excluded, given the large experimental (and theoretical) uncertainties. However, with expected future, significantly reduced uncertainties (experimental of $5 \%$ of the measured value; theoretical of $5 \%$ of the SM value), this observable alone will have the potential to basically rule out the whole AF region, and thus a very broad range of the $\left(m_{0}, m_{1 / 2}\right)$ plane that will for the most part remain beyond the reach of direct sparticle searches at the LHC. Next we showed that DM direct detection search sensitivities expected for future one-tonne detectors provide a complementary and strong tool to test and discriminate between the remaining two high probability regions of the CMSSM: the SC region corresponding to the LSP mass of $\lesssim 450 \mathrm{GeV}$ (and borderline $\sigma_{p}^{\mathrm{SI}}$ ) and the $\sim 1 \mathrm{TeV}$ higgsino region with a much wider range of $\sigma_{p}^{\mathrm{SI}}$. Note also that for $\mu<0$ only the latter case is, for the most part, detectable. This also implies that a DM signal indicative of the SC region would strongly favor the positive sign of $\mu$.

The NUHM presents, unfortunately, a much less clear cut behavior with respect to the interplay of the above observables. In particular this is so because the pseudoscalar mass can be treated as a free parameter of the model and can be adjusted, along with the other parameters, in different ways to yield a good fit to almost all observables. While high probability regions analogous to the CMSSM are also present in the NUHM, and no additional ones, they correspond to different ranges of the parameter space. As a result, unlike in the CMSSM, one can easily identify the AF region with very SM-like $\mathrm{BR}\left(B_{s} \rightarrow \mu^{+} \mu^{-}\right)$. Furthermore, $m_{\chi}$ and $\sigma_{p}^{\mathrm{SI}}$ in the AF region extend to much wider ranges than in the CMSSM. For this reason, in the NUHM it is unlikely to be possible to use future determinations of $\mathrm{BR}\left(B_{s} \rightarrow \mu^{+} \mu^{-}\right)$and $\sigma_{p}^{\mathrm{SI}}$ to convincingly rule out the $A$-funnel, which will also remain for the most part beyond the reach of LHC direct SUSY searches. On the other hand, a measurement of a DM signal in the mass range $500 \mathrm{GeV} \lesssim m_{\chi} \lesssim 800 \mathrm{GeV}$ 
would be a strong indication against the CMSSM where such cases giving a good fit too all data are absent.

\section{Acknowledgments}

We would like to thank Yue-Lin Sming Tsai for helpful discussions throughout. We would also like to thank B. Allanach for useful explanations on the details of different versions of SoftSUSY. L.R. would like to thank G. Isidori, N. Mahmoudi, M. Palutan and B. Pietrzyk for correspondence regarding $\mathrm{BR}\left(B_{s} \rightarrow \mu^{+} \mu^{-}\right)$. This work has been funded in part by the Welcome Programme of the Foundation for Polish Science. K.K. is supported by the EU and MSHE grant N POIG.02.03.00-00-013/09. L.R. is also supported in part by the Polish National Science Centre grant N N202 167440, an STFC consortium grant of Lancaster, Manchester and Sheffield Universities and by the EC 6th Framework Programme MRTNCT-2006-035505. The use of the CIS computer cluster at NCBJ is gratefully acknowledged. L.R. is grateful to the CERN Theory Division for hospitality extended to him during the final stages of this work.

Open Access. This article is distributed under the terms of the Creative Commons Attribution License which permits any use, distribution and reproduction in any medium, provided the original author(s) and source are credited.

\section{References}

[1] CMS collaboration, Observation of a new boson at a mass of $125 \mathrm{GeV}$ with the CMS experiment at the LHC, Phys. Lett. B 716 (2012) 30 [arXiv:1207.7235] [INSPIRE].

[2] ATLAS collaboration, Observation of a new particle in the search for the Standard Model Higgs boson with the ATLAS detector at the LHC, Phys. Lett. B 716 (2012) 1 [arXiv: 1207.7214] [INSPIRE].

[3] CMS collaboration, Combination of standard model Higgs boson searches and measurements of the properties of the new boson with a mass near $125 \mathrm{GeV}$, CMS-PAS-HIG-12-045.

[4] ATLAS collaboration, An update of combined measurements of the new Higgs-like boson with high mass resolution channels, ATLAS-CONF-2012-170 (2012).

[5] LHCB collaboration, First Evidence for the Decay $B_{s}^{0} \rightarrow \mu^{+} \mu^{-}$, Phys. Rev. Lett. 110 (2013) 021801 [arXiv: 1211.2674 ] [INSPIRE].

[6] C.-S. Huang, W. Liao and Q.-S. Yan, The promising process to distinguish supersymmetric models with large tan Beta from the standard model: $B \rightarrow X_{s} \mu^{+} \mu^{-}$, Phys. Rev. D 59 (1999) 011701 [hep-ph/9803460] [INSPIRE].

[7] C. Hamzaoui, M. Pospelov and M. Toharia, Higgs mediated FCNC in supersymmetric models with large tan Beta, Phys. Rev. D 59 (1999) 095005 [hep-ph/9807350] [INSPIRE].

[8] S.R. Choudhury and N. Gaur, Dileptonic decay of B(s) meson in SUSY models with large tan Beta, Phys. Lett. B 451 (1999) 86 [hep-ph/9810307] [InSPIRE].

[9] K. Babu and C.F. Kolda, Higgs mediated $B^{0} \rightarrow \mu^{+} \mu^{-}$in minimal supersymmetry, Phys. Rev. Lett. 84 (2000) 228 [hep-ph/9909476] [InSPIRE]. 
[10] C.-S. Huang, W. Liao, Q.-S. Yan and S.-H. Zhu, $B_{s} \rightarrow \ell^{+} \ell^{-}$in a general 2 HDM and MSSM, Phys. Rev. D 63 (2001) 114021 [Erratum ibid. D 64 (2001) 059902] [hep-ph/0006250] [INSPIRE].

[11] J.R. Ellis, K.A. Olive and V.C. Spanos, On the interpretation of $B_{s} \rightarrow \mu^{+} \mu^{-}$in the CMSSM, Phys. Lett. B 624 (2005) 47 [hep-ph/0504196] [INSPIRE].

[12] LHCB collaboration, Strong constraints on the rare decays $B_{s} \rightarrow \mu^{+} \mu^{-}$and $B^{0} \rightarrow \mu^{+} \mu^{-}$, Phys. Rev. Lett. 108 (2012) 231801 [arXiv:1203.4493] [INSPIRE].

[13] ATLAS collaboration, Search for squarks and gluinos with the ATLAS detector using final states with jets and missing transverse momentum and $5.8 \mathrm{fb}^{-1}$ of $\sqrt{s}=8 \mathrm{TeV}$ proton-proton collision data, ATLAS-CONF-2012-109 (2012).

[14] CMS collaboration, Search for supersymmetry in final states with missing transverse energy and 0, 1, 2, 3, or at least 4 b-quark jets in 8 tev pp collisions using the variable AlphaT, CMS-PAS-SUS-12-028 (2012).

[15] CMS collaboration, Inclusive search for supersymmetry using the razor variables in pp collisions at $\sqrt{s}=7 \mathrm{TeV}$, arXiv:1212.6961 [INSPIRE].

[16] A. Fowlie et al., The CMSSM Favoring New Territories: The Impact of New LHC Limits and a 125 GeV Higgs, Phys. Rev. D 86 (2012) 075010 [arXiv:1206.0264] [InSPIRE].

[17] M. Kadastik, K. Kannike, A. Racioppi and M. Raidal, Implications of the $125 \mathrm{GeV}$ Higgs boson for scalar dark matter and for the CMSSM phenomenology, JHEP 05 (2012) 061 [arXiv: 1112.3647] [INSPIRE].

[18] C. Balázs, A. Buckley, D. Carter, B. Farmer and M. White, Should we still believe in constrained supersymmetry?, arXiv:1205.1568 [INSPIRE].

[19] P. Bechtle et al., Constrained Supersymmetry after two years of LHC data: a global view with Fittino, JHEP 06 (2012) 098 [arXiv: 1204.4199] [INSPIRE].

[20] S. Akula, P. Nath and G. Peim, Implications of the Higgs Boson Discovery for mSUGRA, Phys. Lett. B 717 (2012) 188 [arXiv:1207.1839] [InSPIRE].

[21] C. Beskidt, W. de Boer, D. Kazakov and F. Ratnikov, Constraints on Supersymmetry from LHC data on SUSY searches and Higgs bosons combined with cosmology and direct dark matter searches, Eur. Phys. J. C 72 (2012) 2166 [arXiv:1207.3185] [InSPIRE].

[22] O. Buchmueller et al., The CMSSM and NUHM1 in Light of $7 \mathrm{TeV}$ LHC, $B_{s} \rightarrow \mu^{+} \mu^{-}$and XENON100 Data, Eur. Phys. J. C 72 (2012) 2243 [arXiv:1207.7315] [InSPIRE].

[23] C. Strege et al., Global Fits of the CMSSM and NUHM including the LHC Higgs discovery and new XENON100 constraints, JCAP 04 (2013) 013 [arXiv:1212.2636] [INSPIRE].

[24] M.E. Cabrera, J.A. Casas and R.R. de Austri, The health of SUSY after the Higgs discovery and the XENON100 data, arXiv:1212.4821 [INSPIRE].

[25] M. Citron et al., The End of the CMSSM Coannihilation Strip is Nigh, arXiv:1212.2886 [INSPIRE].

[26] J.R. Ellis, T. Falk and K.A. Olive, Neutralino - Stau coannihilation and the cosmological upper limit on the mass of the lightest supersymmetric particle, Phys. Lett. B 444 (1998) 367 [hep-ph/9810360] [INSPIRE].

[27] M. Drees and M.M. Nojiri, The neutralino relic density in minimal $N=1$ supergravity, Phys. Rev. D 47 (1993) 376 [hep-ph/9207234] [InSPIRE]. 
[28] K.L. Chan, U. Chattopadhyay and P. Nath, Naturalness, weak scale supersymmetry and the prospect for the observation of supersymmetry at the Tevatron and at the CERN LHC, Phys. Rev. D 58 (1998) 096004 [hep-ph/9710473] [INSPIRE].

[29] J.L. Feng, K.T. Matchev and T. Moroi, Focus points and naturalness in supersymmetry, Phys. Rev. D 61 (2000) 075005 [hep-ph/9909334] [INSPIRE].

[30] XENON100 collaboration, E. Aprile et al., Dark Matter Results from 225 Live Days of XENON100 Data, Phys. Rev. Lett. 109 (2012) 181301 [arXiv:1207.5988] [INSPIRE].

[31] L. Roszkowski, R. Ruiz de Austri, R. Trotta, Y.-L.S. Tsai and T.A. Varley, Global fits of the Non-Universal Higgs Model, Phys. Rev. D 83 (2011) 015014 [arXiv:0903.1279] [INSPIRE].

[32] K. Kowalska et al., The Constrained NMSSM with a $125 \mathrm{GeV}$ Higgs boson - A global analysis, arXiv:1211.1693 [INSPIRE].

[33] A. Arbey, M. Battaglia, F. Mahmoudi and D. Martinez Santos, Supersymmetry confronts $B_{s} \rightarrow \mu^{+} \mu^{-}$: Present and future status, Phys. Rev. D 87 (2013) 035026 [arXiv:1212.4887] [INSPIRE].

[34] W. Altmannshofer, M. Carena, N.R. Shah and F. Yu, Indirect Probes of the MSSM after the Higgs Discovery, JHEP 01 (2013) 160 [arXiv:1211.1976] [INSPIRE].

[35] G. Buchalla and A.J. Buras, QCD corrections to rare $K$ and $B$ decays for arbitrary top quark mass, Nucl. Phys. B 400 (1993) 225 [INSPIRE].

[36] M. Misiak and J. Urban, QCD corrections to FCNC decays mediated by $Z$ penguins and $W$ boxes, Phys. Lett. B 451 (1999) 161 [hep-ph/9901278] [INSPIRE].

[37] C. Bobeth, T. Ewerth, F. Krüger and J. Urban, Analysis of neutral Higgs boson contributions to the decays $\bar{B}_{s} \rightarrow \ell^{+} \ell^{-}$and $\bar{B} \rightarrow K \ell^{+} \ell^{-}$, Phys. Rev. D 64 (2001) 074014 [hep-ph/0104284] [INSPIRE].

[38] C. Bobeth, A.J. Buras, F. Krüger and J. Urban, $Q C D$ corrections to $\bar{B} \rightarrow X_{d, s} \nu \bar{\nu}$, $\bar{B}_{d, s} \rightarrow \ell^{+} \ell^{-}, K \rightarrow \pi \nu \bar{\nu}$ and $K_{L} \rightarrow \mu^{+} \mu^{-}$in the MSSM, Nucl. Phys. B 630 (2002) 87 [hep-ph/0112305] [INSPIRE].

[39] A.J. Buras, Minimal flavour violation and beyond: Towards a flavour code for short distance dynamics, Acta Phys. Polon. B 41 (2010) 2487 [arXiv: 1012.1447] [inSPIRE].

[40] F. Mahmoudi, S. Neshatpour and J. Orloff, Supersymmetric constraints from $B_{s} \rightarrow \mu^{+} \mu^{-}$ and $B \rightarrow K * \mu^{+} \mu^{-}$observables, JHEP 08 (2012) 092 [arXiv:1205.1845] [INSPIRE].

[41] K. De Bruyn et al., Probing New Physics via the $B_{s}^{0} \rightarrow \mu^{+} \mu^{-}$Effective Lifetime, Phys. Rev. Lett. 109 (2012) 041801 [arXiv: 1204.1737] [INSPIRE].

[42] LHCB collaboration, Framework TDR for the LHCb Upgrade: Technical Design Report, CERN-LHCC-2012-007

[43] G. Isidori, private communication.

[44] L. Roszkowski, R. Ruiz de Austri and T. Nihei, New cosmological and experimental constraints on the CMSSM, JHEP 08 (2001) 024 [hep-ph/0106334] [INSPIRE].

[45] V.D. Barger, M. Berger and P. Ohmann, The supersymmetric particle spectrum, Phys. Rev. D 49 (1994) 4908 [hep-ph/9311269] [INSPIRE].

[46] A. Fowlie, A. Kalinowski, M. Kazana, L. Roszkowski and Y.S. Tsai, Bayesian Implications of Current LHC and XENON100 Search Limits for the Constrained MSSM, Phys. Rev. D 85 (2012) 075012 [arXiv:1111.6098] [INSPIRE]. 
[47] L. Roszkowski, E.M. Sessolo and Y.-L.S. Tsai, Bayesian Implications of Current LHC Supersymmetry and Dark Matter Detection Searches for the Constrained MSSM, Phys. Rev. D 86 (2012) 095005 [arXiv: 1202.1503] [INSPIRE].

[48] WMAP collaboration, E. Komatsu et al., Seven-Year Wilkinson Microwave Anisotropy Probe (WMAP) Observations: Cosmological Interpretation, Astrophys. J. Suppl. 192 (2011) 18 [arXiv: 1001.4538] [INSPIRE].

[49] Particle Data Group collaboration, J. Beringer et al., Review of Particle Physics (RPP), Phys. Rev. D 86 (2012) 010001 [inSPIRE].

[50] J.P. Miller, E. de Rafael and B.L. Roberts, Muon (g-2): Experiment and theory, Rept. Prog. Phys. 70 (2007) 795 [hep-ph/0703049] [INSPIRE].

[51] http://www.slac.stanford.edu/xorg/hfag/rare/2012/radll/index.html.

[52] Heavy Flavor Averaging Group collaboration, Y. Amhis et al., Averages of B-Hadron, C-Hadron and tau-lepton properties as of early 2012, arXiv:1207.1158 [INSPIRE].

[53] R.R. de Austri, R. Trotta and L. Roszkowski, A Markov chain Monte Carlo analysis of the CMSSM, JHEP 05 (2006) 002 [hep-ph/0602028] [INSPIRE].

[54] S. Heinemeyer, O. Stal and G. Weiglein, Interpreting the LHC Higgs Search Results in the MSSM, Phys. Lett. B 710 (2012) 201 [arXiv:1112.3026] [InSPIRE].

[55] J.R. Ellis, K.A. Olive and C. Savage, Hadronic Uncertainties in the Elastic Scattering of Supersymmetric Dark Matter, Phys. Rev. D 77 (2008) 065026 [arXiv:0801.3656] [INSPIRE].

[56] T. Sjöstrand, S. Mrenna and P.Z. Skands, PYTHIA 6.4 Physics and Manual, JHEP 05 (2006) 026 [hep-ph/0603175] [INSPIRE].

[57] http://physics.ucdavis.edu/ conway/research/software/pgs/pgs4-general.htm.

[58] M.W. Cahill-Rowley, J.L. Hewett, A. Ismail and T.G. Rizzo, More Energy, More Searches, but the pMSSM Lives On, arXiv:1211.1981 [INSPIRE].

[59] https://twiki.cern.ch/twiki/pub/CMSPublic/PhysicsResultsSUS12028/SUS-12-028_ result.pdf.

[60] CMS collaboration, Search for supersymmetry in final states with missing transverse energy and 0, 1, 2, or at least 3 b-quark jets in 7TeV pp collisions using the variable alphaT, JHEP 01 (2013) 077 [arXiv:1210.8115] [INSPIRE].

[61] T. Junk, Confidence level computation for combining searches with small statistics, Nucl. Instrum. Meth. A 434 (1999) 435 [hep-ex/9902006] [INSPIRE].

[62] F. Feroz, M. Hobson and M. Bridges, MultiNest: an efficient and robust Bayesian inference tool for cosmology and particle physics, Mon. Not. Roy. Astron. Soc. 398 (2009) 1601 [arXiv: 0809.3437] [INSPIRE].

[63] B. Allanach, SOFTSUSY: a program for calculating supersymmetric spectra, Comput. Phys. Commun. 143 (2002) 305 [hep-ph/0104145] [INSPIRE].

[64] A. Arbey and F. Mahmoudi, SuperIso Relic: A Program for calculating relic density and flavor physics observables in Supersymmetry, Comput. Phys. Commun. 181 (2010) 1277 [arXiv: 0906.0369] [INSPIRE].

[65] S. Heinemeyer, W. Hollik and G. Weiglein, FeynHiggs: A Program for the calculation of the masses of the neutral CP even Higgs bosons in the MSSM, Comput. Phys. Commun. 124 (2000) 76 [hep-ph/9812320] [INSPIRE]. 
[66] G. Bélanger, F. Boudjema, A. Pukhov and A. Semenov, MicrOMEGAs 2.0: A Program to calculate the relic density of dark matter in a generic model, Comput. Phys. Commun. 176 (2007) 367 [hep-ph/0607059] [INSPIRE].

[67] R. Trotta, F. Feroz, M.P. Hobson, L. Roszkowski and R. Ruiz de Austri, The impact of priors and observables on parameter inferences in the Constrained MSSM, JHEP 12 (2008) 024 [arXiv:0809.3792] [INSPIRE].

[68] G.F. Giudice and A. Strumia, Probing High-Scale and Split Supersymmetry with Higgs Mass Measurements, Nucl. Phys. B 858 (2012) 63 [arXiv:1108.6077] [InSPIRE].

[69] G. Bélanger, S. Kraml and A. Pukhov, Comparison of SUSY spectrum calculations and impact on the relic density constraints from WMAP, Phys. Rev. D 72 (2005) 015003 [hep-ph/0502079] [INSPIRE].

[70] CMS collaboration, Discovery potential for supersymmetry in CMS, J. Phys. G 28 (2002) 469 [hep-ph/9806366] [INSPIRE].

[71] XENON1T collaboration, E. Aprile, The XENON1T Dark Matter Search Experiment, arXiv:1206.6288 [INSPIRE]. 